\title{
GROUP REPRESENTATIONS AND LATTICES
}

\author{
BENEDICT H. GROSS
}

This paper began as an attempt to understand the Euclidean lattices that were recently constructed (using the theory of elliptic curves) by Elkies [E] and Shioda [Sh1, Sh2], from the point of view of group representations. The main idea appears in a note of Thompson [Th2]: if one makes strong irreducibility hypotheses on a rational representation $V$ of a finite group $G$, then the $G$-stable Euclidean lattices in $V$ are severely restricted. Unfortunately, these hypotheses are rarely satisfied when $V$ is absolutely irreducible over $\mathbf{Q}$. But there are many examples where the ring $\operatorname{End}_{G}(V)$ is an imaginary quadratic field or a definite quaternion algebra. These representations allow us to construct some of the Mordell-Weil lattices considered by Elkies and Shioda, as well as some interesting even unimodular lattices that do not seem to come from the theory of elliptic curves.

In $\S 1$ we discuss lattices and Hermitian forms on $V$, and in $\S \S 2-4$ the strong irreducibility hypotheses we wish to make. In $\S 5$ we show how our hypotheses imply the existence of a finite number (up to isomorphism) of Euclidean $\mathbf{Z}[G]$ lattices $L$ in $V$ with $\operatorname{End}_{G}(L)$ a maximal order in $\operatorname{End}_{G}(V)$. We give some examples with $\operatorname{dim} V \leq 8$ in $\S 6$, and in $\S \S 7-9$ discuss the invariants of $L$, such as the dual lattice and theta function. The rest of the paper is devoted to examples: in most, $G$ is a finite group of Lie type and $V$ is obtained as an irreducible summand of the Weil representation of $G$.

Some of the representation theoretic problems left open by this paper are: to find all examples of pairs $(G, V)$ satisfying the strong irreducibility hypotheses of $\S \S 2-4$, and to determine the invariants (shortest nonzero vector, theta function, Thompson series, ...) of the $G$-lattices $L$ so effortlessly constructed inside $V$.

\section{LATTICES AND HERMITIAN FORMS}

In this section, we establish the notation that will be used throughout the paper. Let $G$ be a finite group of order $g$. Elements of $G$ will be denoted $s, t, \ldots$. Let $V$ be a finite-dimensional rational vector space that affords a linear representation of $G$ over $\mathbf{Q}$. We view elements of $G$ as linear operators acting on the right of $V$, and so have the formula: $v^{s t}=\left(v^{s}\right)^{t}$ for $v \in V$ and $s, t \in G$. Let $\chi(s)=\operatorname{Trace}_{V}(s)$ be the character of $V$.

Received by the editors April 20, 1990.

1980 Mathematics Subject Classification (1985 Revision). Primary 20C15, 20C20, $11 \mathrm{H} 31$. 
Let $K=\operatorname{End}_{G}(V)$ be the commuting algebra of $G$ in $\operatorname{End}(V)$, which consists of all Q-linear transformations $\alpha: V \rightarrow V$ which satisfy $\alpha\left(v^{s}\right)=\alpha(v)^{s}$ for all $v \in V$ and $s \in G$. We view $V$ as a left $K$-module. Recall that an order $R$ in the Q-algebra $K$ is a subring that is free of rank $=\operatorname{dim}_{\mathbf{Q}}(K)$ as a Z-module and satisfies $R \otimes \mathbf{Q}=K$. By definition, a lattice $L$ in $V$ is a free Z-submodule of $\operatorname{rank}=\operatorname{dim}_{\mathbf{Q}}(V)$ which satisfies $L \otimes \mathbf{Q}=V$. We say that $L$ is an $R G$-lattice in $V$ if it is stable under left multiplication by $R$ and the right action of $G$.

If $L_{0}$ is any lattice in $V$, then

$$
L=\sum_{s \in G} R L_{0} s=R \cdot L_{0} \cdot \mathbf{Z}[G]
$$

is an $R G$-lattice. By an ideal $\mathfrak{a}$ of $R$ we will always mean a two-sided, nonzero ideal; we say $\mathfrak{a}$ is maximal if there are no two-sided ideals $\mathfrak{b}$ which satisfy $\mathfrak{a} \varsubsetneqq \mathfrak{b} \varsubsetneqq R$. If $L$ is an $R G$-lattice, then so is $\mathfrak{a} L=\{\alpha v: \alpha \in \mathfrak{a}, v \in L\}$.

Let $\alpha \mapsto \bar{\alpha}$ be a fixed anti-involution of the Q-algebra $K$ (so $\overline{\alpha+\beta}=\bar{\alpha}+\bar{\beta}$ and $\overline{\alpha \beta}=\bar{\beta} \bar{\alpha})$. A $K$-Hermitian form $\varphi$ on $V$ is a Q-bilinear pairing $\varphi$ : $V \times V \rightarrow K$ which satisfies (cf. [MH, Appendix 2])

$$
\left\{\begin{aligned}
\varphi(\alpha v, \beta w) & =\alpha \varphi(v, w) \bar{\beta}, \\
\varphi(w, v) & =\overline{\varphi(v, w)} .
\end{aligned}\right.
$$

We say $\varphi$ is nondegenerate if the map $V \rightarrow \operatorname{Hom}_{K}(V, K)$ given by $w \mapsto$ $f_{w}(v)=\varphi(v, w)$ is an isomorphism of $\mathbf{Q}$-vector spaces. We say that $\varphi$ is $G$-invariant if $\varphi\left(v^{s}, w^{s}\right)=\varphi(v, w)$ for all $v, w \in V$ and all $s \in G$. If $\varphi_{0}$ is a $K$-Hermitian form on $V$, the sum

$$
\varphi(v, w)=\sum_{s \in G} \varphi_{0}\left(v^{s}, w^{s}\right)
$$

is $G$-invariant.

Let $R$ be an order in $K$ with $R=\bar{R}$, and let $L$ be an $R G$-lattice in $V$. If $\varphi$ is a nondegenerate, $G$-invariant Hermitian form on $V$, the Hermitian dual

$$
L_{\varphi}^{\prime}=\{w \in V: \varphi(L, w) \in R\}=\{v \in V: \varphi(v, L) \in R\}
$$

is also an $R G$-lattice in $V$.

\section{IRREDUCIBILITY OVER $R$}

In this section, we consider the representation of $G$ on the real vector space $V \otimes \mathbf{R}$. Recall that a symmetric bilinear form on $V \otimes \mathbf{R}$ is a bilinear pairing $\langle\rangle:,(V \otimes \mathbf{R}) \times(V \otimes \mathbf{R}) \rightarrow \mathbf{R}$ that satisfies $\langle v, w\rangle=\langle w, v\rangle$. This form is $G$-invariant if $\left\langle v^{s}, w^{s}\right\rangle=\langle v, w\rangle$ for all $s \in G$, and positive definite if $\langle v, v\rangle \geq 0$, with equality holding only for $v=0$.

The space of $G$-invariant symmetric bilinear forms on $V$ is finite dimensional over $\mathbf{R}$, and the positive-definite forms give an $\mathbf{R}_{+}^{*}$-cone in this vector space. This cone is always nonempty: if $\langle,\rangle_{0}$ is any positive-definite symmetric bilinear form on $V$, the sum $\langle v, w\rangle=\sum_{s \in G}\left\langle v^{s}, w^{s}\right\rangle_{0}$ is positive definite and $G$-invariant. 
Proposition 2.1. The following are all equivalent.

(1) The representation of $G$ on $V \otimes \mathbf{R}$ is irreducible (over $\mathbf{R}$ ).

(2) The $\mathbf{R}$-algebra $K \otimes \mathbf{R}$ is a division ring.

(3) We have $\frac{1}{g} \sum_{s \in G} \frac{1}{2}\left\{\chi(s)^{2}+\chi\left(s^{2}\right)\right\}=1$.

(4) The space of $G$-invariant symmetric bilinear forms on $V \otimes \mathbf{R}$ has dimension $=1$.

(5) The cone of positive-definite G-invariant symmetric bilinear forms on $V \otimes \mathbf{R}$ is a principal homogeneous space for $\mathbf{R}_{+}^{*}$.

Proof. (1) $\Rightarrow(2)$ by Schur's lemma. If $K \otimes \mathbf{R}$ is a division ring, it is isomorphic to $\mathbf{R}, \mathbf{C}$, or $\mathbf{H}$ by Frobenius' theorem. In the first case $\chi=\psi$ is an irreducible complex character of $G$, in the second $\chi=\psi+\bar{\psi}$ where $\psi$ is irreducible and is not equal to its conjugate, in the third $\chi=2 \psi$ where $\psi$ is irreducible and real valued. By the orthogonality relations on irreducible characters over $\mathbf{C}$, we find $\frac{1}{g} \sum_{s \in G} \chi(s)^{2}=\langle\chi, \chi\rangle=1,2$, or 4 in the three cases. But by the criterion of Frobenius-Schur (cf. $[S 3,13.2]), \frac{1}{g} \sum_{s \in G} \chi\left(s^{2}\right)=1,0$, or -2 . Hence, adding these sums and dividing by 2 , we see that $(2) \Rightarrow(3)$.

The sum in (3) is the dimension of the fixed space in the representation $\operatorname{Sym}^{2} V$, with character $\frac{1}{2}\left\{\chi(s)^{2}+\chi\left(s^{2}\right)\right\}$. This is the same as the dimension of the fixed space in the dual representation $\operatorname{Sym}^{2} V^{*}$, or equivalently, the $G$-invariant symmetric bilinear forms on $V$ (or $V \otimes \mathbf{R}$ ). Hence $(3) \Rightarrow(4)$. Clearly $(4) \Rightarrow(5)$, as the cone of $G$-invariant positive-definite forms is always nonempty. Finally, if $V \otimes \mathbf{R}=W \oplus U$ is not irreducible, we have at least a twodimensional cone of positive-definite bilinear forms on $V$ that are $G$-invariant: $a\langle,\rangle_{W}+b\langle,\rangle_{U}$, where $a, b>0$ and $\langle,\rangle_{W}$ and $\langle,\rangle_{U}$ are positive-definite $G$-invariant forms on $W$ and $U$ respectively. Hence (5) $\Rightarrow(1)$.

Corollary 2.2. Assume that the representation of $G$ on $V \otimes \mathbf{R}$ is irreducible. Then the $\mathbf{Q}$-algebra $K=\operatorname{End}_{G}(V)$ is isomorphic either to $\mathbf{Q}$, an imaginary quadratic field, or a definite quaternion algebra.

Proof. These are the only $\mathbf{Q}$-algebras $K$ with $K \otimes \mathbf{R}$ a division ring.

We henceforth assume $V \otimes \mathbf{R}$ is irreducible, so $K$ is described by Corollary 2.2. Let $\alpha \mapsto \bar{\alpha}$ be the canonical anti-involution of $K$ : this is the identity for $K=\mathbf{Q}$, the generator of $\operatorname{Gal}(K / \mathbf{Q})$ when $K$ is quadratic, and induces the Galois automorphism of any quadratic subfield when $K$ is quaternionic. In all cases, the subfield of $K$ fixed by the anti-involution is equal to $\mathbf{Q}$, and $\alpha \bar{\alpha} \geq 0$ with equality holding only for $\alpha=0$. We say a $K$-Hermitian form $\varphi$ on $V$ is positive definite if $\varphi(v, v) \geq 0$, with equality holding only for $v=0$.

Proposition 2.3. If $V \otimes \mathbf{R}$ is irreducible, the cone of positive-definite G-invariant $K$-Hermitian forms $\varphi: V \times V \rightarrow K$ is a principal homogeneous space for $\mathbf{Q}_{+}^{*}$.

Proof. A positive-definite $G$-invariant form $\varphi$ exists on $V$, by the standard averaging argument. Hence we must show that if $\varphi$ and $\varphi^{\prime}$ are two such 
forms, there is an element $\alpha \in \mathbf{Q}_{+}^{*}$ such that $\varphi^{\prime}=\alpha \varphi$. Both $\varphi$ and $\varphi^{\prime}$ give $G$-isomorphisms from $V$ to $\operatorname{Hom}_{K}(V, K)$, so by Schur's lemma we have $\varphi(v, w)=\varphi^{\prime}(v, \alpha w)$ for some $\alpha \in K^{*}=\operatorname{Aut}_{G}(V)$. Using the fact that the forms are both Hermitian, we find $\varphi^{\prime}(v, \alpha w)=\varphi^{\prime}(\alpha v, w)$ which implies that $\alpha \in \mathbf{Q}^{*}$. Hence $\varphi^{\prime}=\alpha \varphi$, and we must have $\alpha>0$ if both forms are positive definite.

If $\varphi$ is a Hermitian, $G$-invariant, positive-definite form on $V$, we define

$$
\langle v, w\rangle=\operatorname{Tr}_{K / \mathbf{Q}} \varphi(v, w)= \begin{cases}\varphi(v, w) & K=\mathbf{Q}, \\ \varphi(v, w)+\varphi(w, v) & K \neq \mathbf{Q} .\end{cases}
$$

This is a positive-definite $G$-invariant symmetric bilinear form on $V$, and the map $\varphi \mapsto\langle$,$\rangle is a bijection between the two \mathbf{Q}_{+}^{*}$ cones of dimension one. If $\delta \in K$ satisfies $\operatorname{Tr}_{K / \mathbf{Q}}(\delta)=0$, the form $\operatorname{Tr}_{K / \mathbf{Q}}(v, \delta w)$ is $G$-invariant and alternating. We obtain, in this manner, the vector space of $G$-invariant alternating forms on $V$, which has dimension $=\operatorname{dim}_{\mathbf{Q}}(K)-1=0,1$, or 3 .

\section{IRREDUCIBILITY (MODULO $\mathfrak{p}$ )}

We continue with the assumptions of the previous section, so $V \otimes \mathbf{R}$ is irreducible, and $K$ is either $\mathbf{Q}$, imaginary quadratic, or a definite quaternion algebra. Let $R$ be a maximal order in $K$, which is unique except in the quaternionic case. If $\mathfrak{p}$ is a maximal (two-sided) ideal of $R$, then $\mathfrak{p}$ contains $p R$ for a unique rational prime $p$. In fact, either $\mathfrak{p}=p R$ or $\mathfrak{p} \cdot \overline{\mathfrak{p}}=p R$. If $\mathfrak{p}^{2}=p R$ we say that $\mathfrak{p}$ is a ramified prime; the set of ramified primes is finite, and nonempty when $K \neq \mathbf{Q}$. For any maximal $\mathfrak{p}$ the quotient ring $k_{\mathfrak{p}}=R / \mathfrak{p} R$ is a simple $\mathbf{Z} / p \mathbf{Z}$-algebra, isomorphic either to $\mathbf{F}_{p}, \mathbf{F}_{p^{2}}$ or the ring $M_{2}\left(\mathbf{F}_{p}\right)$ of $2 \times 2$ matrices over the prime field.

Let $L$ be an $R G$-lattice in $V$, and $\mathfrak{p}$ a maximal ideal of $R$. We define the reduced representation:

$$
V_{\mathfrak{p}}=L / \mathfrak{p} L \quad \text { of } G \text { over } \quad k_{\mathfrak{p}}=R / \mathfrak{p} R .
$$

This depends on the choice of lattice $L$, although it does not appear in our notation. We say $V_{\mathfrak{p}}$ is irreducible over $k_{\mathfrak{p}}$ if there are no nontrivial $k_{\mathfrak{p}}$-submodules that are stable under the right action of $G$.

Proposition 3.2. The following are equivalent.

(1) For all maximal ideals $\mathfrak{p}$ of $R$ the representation $V_{\mathfrak{p}}$ of $G$ is irreducible over $k_{\mathfrak{p}}=R / \mathfrak{p} R$.

(2) Every $R G$-lattice in $V$ has the form $\mathfrak{a} L$, where $\mathfrak{a}$ is a fractional ideal of $R$.

Proof. Let $W_{\mathfrak{p}}$ be a nontrivial $k_{\mathfrak{p}} G$-submodule of $V_{\mathfrak{p}}$. Then $M=\{v \in L$ : $v(\bmod p L)$ is in $\left.W_{p}\right\}$ is an $R G$-lattice in $V$ with $\mathfrak{p} L \varsubsetneqq M \varsubsetneqq L$. Hence $M$ is not of the form $a L$, and (2) $\Rightarrow(1)$. 
Now assume $V_{\mathfrak{p}}$ is irreducible for all $\mathfrak{p}$, and let $M$ be an $R G$-lattice in $V$. Define $L_{p}=L \otimes \mathbf{Z}_{p}, R_{p}=R \otimes \mathbf{Z}_{p}, K_{p}=K \otimes \mathbf{Q}_{p}$, and $V_{p}=V \otimes \mathbf{Q}_{p}$. We will show that any $R_{p} G$ lattice $M_{p}=M \otimes Z_{p}$ in $V_{p}$ has the form $M_{p}=\alpha_{p} L_{p}$ with $\alpha_{p}$ an element of $K_{p}^{*}$ which normalizes $R_{p}$. The global result follows from this, taking $a$ to be the fractional ideal $K \cap \prod_{p} \alpha_{p} R_{p}$, so $M=\mathfrak{a} L$.

First assume $K_{p}$ is a division ring, so $R_{p}$ is a local ring with maximal ideal $\mathfrak{p}=\pi R_{p}$. If $M_{p}$ is an $R_{p} G$ lattice we may assume, after scaling by a power of $\pi$, that $M_{p}$ is contained in $L_{p}$ but not in $\pi L_{p}$. Hence the image of $M_{p}$ in $V_{\mathrm{p}}=L_{p} / \pi L_{p}$ is nonzero. By the hypothesis that $V_{\mathrm{p}}$ is irreducible, the image must be equal to $L_{p} / \pi L_{p}$, so $M_{p}+\pi L_{p}=L_{p}$. Nakayama's lemma (applied to $L_{p} / M_{p}$ ) shows that $M_{p}=L_{p}$. Hence any $R_{p} G$-lattice $M_{p}$ has the form $\pi^{n} L_{p}$ for some $n \in \mathbf{Z}$.

Next assume that $K_{p}=\mathbf{Q}_{p} \times \mathbf{Q}_{p}$, so $p R=\mathfrak{p} \overline{\mathfrak{p}}$ with $\mathfrak{p} \neq \overline{\mathfrak{p}}$. Then $V_{p}=W_{\mathfrak{p}} \oplus W_{\overline{\mathfrak{p}}}$ is the sum of nonisomorphic irreducible representations of $G$ over $\mathbf{Q}_{p}$, with $W_{\bar{p}} \simeq \operatorname{Hom}\left(W_{\mathfrak{p}}, \mathbf{Q}_{p}\right)$. We have a decomposition $L_{p}=N_{\mathfrak{p}} \oplus N_{\bar{p}}$, where $N_{\mathfrak{p}}$ and $N_{\bar{p}}$ are $\mathbf{Z}_{p} G$-lattices in $W_{\mathfrak{p}}$ and $W_{\overline{\mathfrak{p}}}$. The irreducibility of $V_{\mathfrak{p}} \simeq N_{\mathfrak{p}} / p N_{\mathfrak{p}}$ and $V_{\bar{p}} \simeq N_{\bar{p}} / p N_{\bar{p}}$ implies, by the argument above, that any $R_{p} G$-lattice $M_{p}$ in $V_{p}$ has the form $p^{a} N_{p} \oplus p^{b} N_{\bar{p}}=\alpha_{p} L_{p}$ with $\alpha_{p}=\left(p^{a}, p^{b}\right)$ in $K_{p}^{*}$.

Finally, assume $K \otimes \mathbf{Q}_{p}$ is isomorphic to $M_{2}\left(\mathbf{Q}_{p}\right)$; we fix an isomorphism taking $R_{p}$ to the standard maximal order $M_{2}\left(Z_{p}\right)$ (this is possible, as all maximal orders are locally conjugate). The isomorphism $K_{p} \simeq M_{2}\left(\mathbf{Q}_{p}\right)$ gives a decomposition $V_{p}=W_{p} \oplus W_{p}$, where $W_{p}$ is irreducible over $\mathbf{Q}_{p}$, and $L_{p}=N_{p} \oplus N_{p}$ with $N_{p}$ a $\mathbf{Z}_{p} G$-lattice in $W_{p}$. The irreducibility of $V_{\mathfrak{p}}=N_{p} / p N_{p} \oplus N_{p} / p N_{p}$ over $k_{\mathfrak{p}}=M_{2}\left(\mathbf{F}_{p}\right)$ implies that $N_{p} / p N_{p}$ is an irreducible $G$-module over $\mathbf{F}_{p}$. Hence, by the above, any $\mathbf{Z}_{p} G$ stable lattice in $W_{p}$ has the form $p^{a} N_{p}$, and any $R_{p} G$ stable lattice in $V_{p}$ has the form $p^{a} N_{p} \oplus p^{a} N_{p}=p^{a} L_{p}$.

\section{GLOBAL IRREDUCIBILITY AND CHARACTERS}

We henceforth only consider rational representations $V$ of $G$ that satisfy the conditions in Propositions 2.1 and 3.2; such representations will be called "globally irreducible."

An interesting problem is to determine all globally irreducible rational representations $V$ of a finite group $G$. Of course, any one-dimensional representation over $\mathbf{Q}$ is globally irreducible, but there may be others. In this section, we suppose that we are given the table of all irreducible complex characters $\psi$ of $G$, as well as the table of its irreducible modular characters $\rho$ over an algebraically closed field of characteristic $p$, for all rational primes $p$. We wish to find some simple conditions that imply the existence of a globally irreducible rational representation $V$ for $G$.

Lemma 4.1. Let $\psi$ be an irreducible complex character of $G$, and let $\mathfrak{p}$ be a finite place of the number field $\mathbf{Q}(\psi)$. If the reduction of $\psi(\bmod \mathfrak{p})$ is an absolutely irreducible Brauer character $\rho$ of $G$, then a representation $W$ of $G$ with character $\psi$ can be defined over the completion $\mathbf{Q}(\psi)_{\mathfrak{p}}$. 
Proof. The obstruction to defining $W$ is the Schur index $e_{\mathrm{p}}(\psi)$. Let $\rho$ be any absolutely irreducible Brauer character of $G$ and $\mathbf{F}_{\rho}$ the finite field generated by its values over $\mathbf{F}_{p}$. Then $e_{\mathfrak{p}}(\psi)$ divides the product $\operatorname{deg}\left(\mathbf{F}_{\rho} \cdot \mathbf{F}_{\mathfrak{p}}: \mathbf{F}_{\mathfrak{p}}\right) \cdot \operatorname{mult}_{\rho}(\psi)$ [F2, Theorem IV.9.3]. If $\psi \equiv \rho$ is irreducible, this product is $=1$, so $e_{\mathfrak{p}}(\psi)=$ 1 .

Proposition 4.2. Let $\psi$ be an irreducible complex character of $G$. (1) Assume $\mathbf{Q}(\psi)=\mathbf{Q}$ and $\psi \equiv \rho(\bmod p)$ is an absolutely irreducible Brauer character of $G$ for all rational primes $p$. Then $\chi=\psi$ is the character of a globally irreducible rational representation $V$ of $G$.

(2) Assume $\mathbf{Q}(\psi)$ is an imaginary quadratic field, and $\psi \equiv \rho(\bmod \mathfrak{p})$ is an absolutely irreducible Brauer character of $G$ for all primes $\mathfrak{p}$ of $\mathbf{Q}(\psi)$. Then $\chi=\psi+\bar{\psi}$ is the character of a globally irreducible rational representation $V$ of $G$.

(3) Assume $\mathbf{Q}(\psi)=\mathbf{Q}$, that $\frac{1}{g} \sum_{s \in G} \psi\left(s^{2}\right)=-1$, and that, for all rational primes $p$, either $\psi \equiv \rho(\bmod p)$ is either absolutely irreducible, or $\psi \equiv \rho+\rho^{p}$ $(\bmod p)$ where $\rho$ is an absolutely irreducible Brauer character with $\mathbf{F}_{\rho}=\mathbf{F}_{p^{2}}$. Then $\chi=2 \psi$ is the character of a globally irreducible rational representation $V$ of $G$.

Proof. (1) By Lemma 4.1 we have $e_{p}(\psi)=1$ for all finite $p$. Hence $e(\psi)=1$, as the obstruction would be a quaternion algebra split at all finite primes, and therefore globally split. Hence $\chi=\psi$ is the character of a rational representation $V$. It is clearly globally irreducible with $\operatorname{End}_{G}(V)=\mathbf{Q}$.

(2) The same argument shows that $\psi$ is the character of a representation $W$ of $G$ over $\mathbf{Q}(\psi)=K$. Viewing $W$ as a rational representation $V$ of $G$ with $\operatorname{End}_{G}(V)=K$, it is clear that $V$ is globally irreducible.

(3) The condition $\frac{1}{g} \sum \psi\left(s^{2}\right)=-1$ implies that $e_{\infty}(\psi)=2$ [Se, Proposition 39]. But when $\mathbf{Q}(\psi)=\mathbf{Q}$ we have $e(\psi)=1$ or 2 by the Brauer-Speiser theorem (cf. [F1, §2]), so $e(\psi)=2$ and $\chi=2 \psi$ is the character of a rational representation $V$ of $G$ with $\operatorname{End}_{G}(V)=K$ a definite quaternion algebra. $V$ is clearly globally irreducible.

Note 4.3. In case (3), the primes $\mathfrak{p}$ which ramify in $K$ (where $e_{\mathfrak{p}}(\psi)=2$ ) are among those where the reduction $\psi \equiv \rho+\rho^{p}(\bmod p)$ is not absolutely irreducible. Indeed, if $\mathfrak{p}^{2}=p R$, the character of $V_{\mathrm{p}}$ over $k_{\mathrm{p}}$ is equal to $\rho$ (after choosing a suitable identification of $k_{\mathfrak{p}}$ with $\mathbf{F}_{p^{2}}$ ).

Note 4.4. The absolute irreducibility of $\psi(\bmod \mathfrak{p})$ is automatic if the residue characteristic $p$ does not divide the order of $G$. So there are only finitely many conditions to check in Proposition 4.2.

\section{Hermitian lattices}

Let $V$ be a globally irreducible representation of $G$, and let $R$ be a maximal order in $K=\operatorname{End}_{G}(V)$. Let $L$ be an $R G$-lattice in $V$, and let $\varphi$ be a positive 
definite $G$-invariant Hermitian form on $V$. Since $\varphi(v, v)>0$ for $v \neq 0$, the form $\varphi$ is nondegenerate and we may define the dual

$$
L_{\varphi}^{\prime}=\{v \in V: \varphi(v, L) \in R\} .
$$

Then $L_{\varphi}^{\prime}$ is an $R G$-lattice, so by Proposition $3.2 L_{\varphi}^{\prime}=\mathfrak{a} L$ for a fractional ideal $\mathfrak{a}$ of $R$ which depends on $\varphi$. We now show that $\varphi$ may be chosen so that $\mathfrak{a}$ is the product of certain ramified primes $\mathfrak{p}$ in $R$.

Proposition 5.2. Let $L$ be an RG-lattice in $V$. Then there is a $G$-invariant, positive definite Hermitian form $\varphi$ on $V$ such that

$$
L_{\varphi}^{\prime}=\left(\prod_{\mathfrak{p} \in S} \mathfrak{p}\right) \cdot L,
$$

where $S$ is a subset of the ramified primes in $R$. The subset $S$ is determined uniquely by the representation $V$, and the form $\varphi$ is determined uniquely by the $R G$-lattice $L$.

Proof. Let $\varphi_{0}$ be any $G$-invariant positive-definite Hermitian form on $V$. Then $L_{\varphi_{0}}^{\prime}=\mathfrak{a} L$ with $\mathfrak{a}$ a fractional ideal; since $\varphi_{0}$ is Hermitian: $\mathfrak{a}=\overline{\mathfrak{a}}$. Hence $\mathfrak{a}=\prod_{\mathfrak{p} \in S} \mathfrak{p} \cdot \alpha \cdot R$, with $S$ a subset of the ramified primes and $\alpha \in \mathbf{Q}_{+}^{*}$. If $\varphi=\alpha \varphi_{0}$ we have $L_{\varphi}^{\prime}=\Pi_{S} \mathfrak{p} \cdot L$ as claimed. Clearly $\varphi$ is uniquely determined by $L$, as the set $S$ and the rational number $\alpha>0$ are uniquely determined by $a$.

If $M=\mathfrak{b} L$ is another $R G$-lattice in $V$, and $\varphi_{m}=\mathbf{N b}^{-1} \cdot \varphi$ with $\mathbf{N b}$ the positive generator of $\mathfrak{b} \cdot \overline{\mathfrak{b}}$, we find $M_{\varphi_{M}}^{\prime}=\prod_{S} \mathfrak{p} \cdot M$. Hence the set $S$ depends only on $V$.

Note 5.3. In fact, the set $S$ in Proposition 5.2 depends only on the local representations $V \otimes \mathbf{Q}_{p}$ at primes $p$ which ramify in $R: p R=\mathfrak{p}^{2}$. In $\S 7$ we will show how the question of whether $\mathfrak{p}$ is in $S$ can often be answered by a consideration of the reduced representation $V_{\mathfrak{p}}$ over $k_{\mathfrak{p}}$.

If $L$ is any $R G$ lattice in $V$, we give it a Hermitian structure using the form $\varphi$ uniquely defined by Proposition 5.2 and a Euclidean structure using the bilinear form $\langle v, w\rangle=\operatorname{Tr}_{K / \mathbf{Q}} \varphi(v, w)$.

Let $C l(R)$ be the two-sided class group of $R$ : this is the quotient of the group of two-sided fractional ideals of $R$ by the subgroup of two-sided principal ideals $\alpha R$, with $\alpha \in K^{*}$ normalizing $R$. The group $C l(R)$ is finite in all cases; when $K=\mathbf{Q}$ it is trivial and when $K$ is quaternionic it is an elementary abelian two-group [V, Chapter III, §5].

Proposition 5.4. The group $C l(R)$ acts simply transitively on the set of isomorphism classes of Euclidean $\mathrm{Z}[G]$-lattices $L$ in $V$ with $\operatorname{End}_{G}(L)=R$.

Proof. We define a simply transitive action of the group of fractional ideals on the set of $R G$-lattices, by $L \mapsto \mathfrak{a} L$. The lattices $L$ and $\mathfrak{a} L$ are isomorphic 
$\mathbf{Z}[G]$-modules if and only if $\mathfrak{a}=\alpha R$ with $\alpha \in K^{*}$, by Schur's lemma. Hence $C l(R)$ acts simply transitively on the isomorphism classes of $\mathbf{Z}[G]$-modules with $\operatorname{End}_{G}(L)=R$. Since the map from $L$ to $\alpha L$ taking $v$ to $\alpha v$ preserves the normalized inner product, we have classified the Euclidean $\mathbf{Z}[G]$-lattices in $V$ with $\operatorname{End}_{G}(L)=R$.

There may be some identification of the Euclidean lattices $L$ so constructed in $V$ if one chooses to ignore the action of $G$. For example,

Lemma 5.5. Let $K=\operatorname{End}_{G}(V)$ be imaginary quadratic. and assume that there is an involution $\tau$ of $V$ which acts $K$-antilinearly ai.l normalizes $G$. Then there is an RG-lattice in $V$ with $\tau(L)=L$. If $\mathfrak{a}$ is an ideal of $K$ we have $\tau(\mathfrak{a} L)=\bar{a} L$ and the map $v \mapsto \tau(v)$ gives an isometry between the Euclidean lattices $\mathfrak{a} L$ and $\overline{\mathrm{a}} L$.

Proof. Let $M$ be an $R G$-lattice. Then $\tau M$ is also an $R G$-lattice, so $\tau M=\mathfrak{b} M$ for an ideal $\mathfrak{b}$ of $K$. Since $\tau^{2}=1$ and $\tau$ is antilinear, $\mathfrak{b} \cdot \overline{\mathfrak{b}}=R$. Hence $\mathfrak{b}=\mathfrak{c} / \overline{\mathfrak{c}}$ and the lattice $L=\mathfrak{c} M$ is fixed by $\tau$. Clearly $\tau(\mathfrak{a} L)=\bar{a} L$, as $\tau$ is $K$-antilinear. Since the bilinear forms on $a L$ and $\bar{a} L$ are both equal to $\mathbf{N a}^{-1} \cdot\langle,\rangle_{L}$, we must show that $\langle v, w\rangle=\langle\tau v, \tau w\rangle$.

The group $G^{\prime}=G \rtimes\langle\tau\rangle$ acts on $V$ with $\operatorname{End}_{G^{\prime}}(V)=\mathbf{Q}$. Hence $G^{\prime}$ stabilizes a one-dimensional space of symmetric bilinear forms, which is exactly the space stabilized by the subgroup $G$. Hence $\tau$ is an isometry of $\langle,\rangle_{L}$.

\section{EXAMPLES IN LOW DIMENSION}

We now give some simple examples of globally irreducible faithful representations $V$ of finite groups $G$. In each case the class group of $R$ is trivial (and all maximal orders are conjugate in $K$ ) so we obtain a unique $\mathbf{Z}[G]$ lattice $L$ in $V$ with $\operatorname{End}_{G}(L)=R$.

The simplest examples are obtained by taking $V=K$ and $G$ a subgroup of $R^{*}$ which does not lie in a proper Q-subspace of $K$. Unfortunately, this puts a severe restriction on the field $K$; the only possibilities are $K=$ $\mathbf{Q}, \mathbf{Q}\left(\mu_{4}\right), \mathbf{Q}\left(\mu_{6}\right)$ and the definite quaternion algebras ramified at $\{2, \infty\}$ and $\{3, \infty\}$. When $K=\mathbf{Q}$ the group $G$ has order 1 or 2 and $L$ is the $A_{1}$-root lattice. When $K=\mathbf{Q}\left(\mu_{4}\right)$ the group $G$ is cyclic of order 4 and $L$ is the $A_{1} \times A_{1}$-root lattice. When $K=\mathbf{Q}\left(\mu_{6}\right)$ the group $G$ is cyclic of order 3 or 6 and $L$ is the $A_{2}$-root lattice. When $K$ is the quaternion algebra ramified at $\{2, \infty\}$ we have $R^{*} \simeq \mathrm{SL}_{2}(3)$ of order 24 , which contains the quaternion group of order 8 as a normal subgroup. Both act globally irreducibly on $K$, and the stable lattice is the $D_{4}$-root lattice of Hurwitz quaternions. When $K$ is the quaternion algebra ramified at $\{3, \infty\}$ we have $G=R^{*}=\widetilde{S}_{3}$ of order 12 . The stable lattice $L$ is the $A_{2} \times A_{2}$-root lattice.

Examples where $\operatorname{dim}_{K} V>1$ are harder to find. For example, when $K=\mathbf{Q}$ we will see that any such $V$ has $\operatorname{dim} V \equiv 0(\bmod 8)($ Proposition 7.4$)$. The simplest example in this case is to take $G=$ the Weyl group of $E_{8}$, of order 
$696729600=2^{14} 3^{5} 5^{2} 7$ and $V$ its eight-dimensional reflection representation! The stable lattice $L$ is the $E_{8}$-root lattice.

When $K=\mathbf{Q}(\sqrt{-2})$ there is an example with $\operatorname{dim}_{K} V=2$. Let $G=$ $\mathrm{GL}_{2}(3)=\widetilde{S}_{4}$, which has order $48=2^{4} \cdot 3$. Let $\psi$ be the character of a discrete series representation of $\mathrm{GL}_{2}(3)$, corresponding to a character of order 8 of the nonsplit torus, that satisfies $\mathbf{Q}(\psi)=K$. This has degree 2; its reduction $(\bmod \sqrt{-2})$ is the irreducible representation $\rho$ of degree 2 of $S_{4}$, and its reduction modulo the two primes dividing 3 is the standard two-dimensional representation of $\mathrm{GL}_{2}(3)$ and its dual. Hence $\chi=\psi+\bar{\psi}$ is the character of a globally irreducible $V$ of dimension four by Proposition 4.2. The Hermitian form $\varphi$ has matrix

$$
\varphi=\left(\begin{array}{ll}
2 & \alpha \\
\bar{\alpha} & 2
\end{array}\right) \quad \text { with } \alpha=1+\sqrt{-2}
$$

with respect to a suitable $R$-basis of $L=L^{\prime}$. The corresponding Euclidean lattice is (a scaling of) the $D_{4}$-lattice. This Hermitian lattice arises from the theta polarization of the Jacobian of the complex curve $X$ of genus 2 with equation $y^{2}=x^{5}-x$; we have $\operatorname{Aut}_{\mathrm{C}}(X)=G$.

When $K=\mathbf{Q}(\sqrt{-7})$ there is an example with $\operatorname{dim}_{K} V=3$. Let $G=$ $\operatorname{PSL}_{2}(7)=\operatorname{SL}_{3}(2)$, which has order $168=2^{3} \cdot 3 \cdot 7$. Let $\psi$ be the character of a $\frac{1}{2}$-discrete series representation of $\operatorname{PSL}_{2}(7)$, corresponding to the quadratic character of the nonsplit torus, which satisfies $\mathbf{Q}(\psi)=K$. This has degree 3 and its reduction is absolutely irreducible at all primes. Indeed, if $\rho \neq 1$ is an irreducible Brauer character of $G$ then $\operatorname{dim} \rho \geq 3$. At the two primes dividing 2 one obtains the standard three-dimensional representation of $\mathrm{SL}_{3}(2)$ and its dual; $(\bmod \sqrt{-7})$ one obtains the adjoint representation of $\operatorname{PSL}_{2}(7)$. Hence $\chi=\psi+\bar{\psi}$ is the character of a globally irreducible representation $V$ of dimension six by Proposition 4.2. The Hermitian form $\varphi$ has the matrix

$$
\varphi=\left(\begin{array}{ccc}
2 & \alpha & \bar{\alpha} \\
\bar{\alpha} & 2 & -1 \\
\alpha & -1 & 2
\end{array}\right) \quad \text { with } \alpha=\frac{1+\sqrt{-7}}{2}
$$

with respect to a suitable $R$-basis of $L=L^{\prime}$. This Hermitian lattice has 42 short vectors with $\varphi(v, v)=2$, and arises from the theta polarization of the Jacobian of the complex curve $X$ of genus 3 with equation $x y^{3}+y z^{3}+z x^{3}=0$. We have $\operatorname{Aut}_{\mathrm{C}}(X)=G$; for a more detailed description of $L$ due to Serre, see [Ma, pp. 235-236].

\section{RAMIFIED PRIMES}

To make Proposition 5.2 more precise, we wish to determine the subset $S$ of ramified primes which occurs. Let $\mathfrak{p}$ be a ramified prime in $R$. The residue field $R / \mathfrak{p}=k_{\mathfrak{p}}$ is isomorphic to $\mathbf{F}_{p}$ or $\mathbf{F}_{p^{2}}$, and the canonical anti-involution of $R$ gives an involution of $k_{\mathfrak{p}}$ (which is trivial when $k_{\mathfrak{p}}=\mathbf{F}_{p}$ and $x \mapsto x^{p}$ when $\left.k=\mathbf{F}_{p^{2}}\right)$. 
Proposition 7.1. (1) If $\mathfrak{p}$ is not in $S$, there is a nondegenerate $G$-invariant $k_{\mathfrak{p}}$ Hermitian form $\varphi_{\mathrm{p}}: V_{\mathrm{p}} \times V_{\mathrm{p}} \rightarrow k_{\mathrm{p}}$.

(2) If $\mathfrak{p}$ is in $S$, there is a nondegenerate G-invariant, strictly alternating, $k_{\mathfrak{p}}$-bilinear form $\varphi_{\mathfrak{p}}: V_{\mathfrak{p}} \times V_{\mathfrak{p}} \rightarrow k_{\mathfrak{p}}$.

Proof. (1) In this case, the Hermitian for $\varphi$ on $V$ gives a nondegenerate pairing of $R_{p}=R \otimes \mathbf{Z}_{p}$-modules $L_{p} \times L_{p} \rightarrow R_{p}$, with $L_{p}=L \otimes \mathbf{Z}_{p}$. Its reduction (modulo $\mathfrak{p} R_{p}$ ) is the $k_{\mathrm{p}}$-Hermitian form $\varphi_{\mathrm{p}}$.

(2) In this case, let $\pi$ be a uniformizing parameter in $R_{p}$, so $\pi R_{p}=\mathfrak{p} R_{p}$. Then the form $\varphi^{\prime}: L_{p} \times L_{p} \rightarrow R_{p}$ defined by $\varphi^{\prime}(v, w)=\varphi(v, w) \cdot \pi$ is $\mathbf{Z}_{p^{-}}$ bilinear and nondegenerate; its reduction is the form $\varphi_{p}$. Clearly $\varphi_{p}$ is $k_{p}$ linear in the first argument, so it suffices to show that $\varphi_{p}(v, v)=0$, or equivalently, that $\varphi(v, v) \pi$ lies in $\mathfrak{p} R_{p}$. But $\varphi(v, v) \pi$ lies in the intersection of $\mathbf{Q}_{p} \pi$ with $R_{p}$, which is equal to $\mathrm{Z}_{p} \pi \subset \mathfrak{p} R_{p}$.

We can make a simple hypothesis on $V_{\mathfrak{p}}$ which assures that the situations (1) and (2) of Proposition 7.1 cannot occur simultaneously.

Corollary 7.2. Assume that $\operatorname{End}_{\mathrm{F}_{p}[G]}\left(V_{\mathfrak{p}}\right)=k_{\mathfrak{p}}$ and that the residue field $k_{\mathfrak{p}}$ has more than two elements. Then $\mathfrak{p}$ is in $S$ if and only if $V_{\mathfrak{p}}$ is a symplectic representation of $G$ over $k_{\mathfrak{p}}$.

Proof. If $k_{\mathfrak{p}}=\mathbf{F}_{p^{2}}$ our hypothesis implies that $V_{\mathfrak{p}}$ is not isomorphic to its conjugate representation $V_{\mathrm{p}}^{p}$. Hence $V_{\mathrm{p}}$ either has a nondegenerate Hermitian form or a nondegenerate symplectic form, but not both. For one identifies the dual $\operatorname{Hom}_{G}\left(V_{\mathfrak{p}}, k_{\mathfrak{p}}\right)$ with $V_{\mathfrak{p}}^{p}$, and the other identifies the dual with $V_{\mathfrak{p}}$.

If $k_{\mathrm{p}}=\mathbf{F}_{p}$, our hypothesis implies that the space of bilinear forms on $V$ has dimension $=1=\operatorname{dim} \operatorname{Hom}_{G}\left(V_{\mathrm{p}}, V_{\mathrm{p}}\right)$. If $p>2$, we cannot have both nondegenerate symmetric and alternating forms on $V_{p}$.

Note 7.3. When $k_{\mathfrak{p}}=\mathbf{F}_{2}$, the symmetric form $\varphi_{\mathfrak{p}}$ on $V_{\mathfrak{p}}$ constructed in (1) is strictly alternating once $\operatorname{dim} V_{p}>1$. For $l(v)=\varphi_{p}(v, v)$ gives a $G$-invariant linear form on $V_{p}$.

We end this section with some elementary remarks on the dimension of $V$ over $\mathbf{Q}$. If $V$ has dimension one then $K=\mathbf{Q}$ and $L$ is isomorphic to $\mathbf{Z}$.

Proposition 7.4 [Th2; S3, Example 15.4]. (1) If $K=\mathbf{Q}$ and $\operatorname{dim} V>1$ then any $\mathbf{Z}[G]$-lattice $L$ in $V$ is even and unimodular. Hence $\operatorname{dim} V \equiv 0(\bmod 8)$.

(2) If $K$ is quadratic, then $\operatorname{dim} V \equiv 0(\bmod 2)$. If $\operatorname{dim} V \equiv 2(\bmod 4)$, then $S$ is empty and $L_{\varphi}^{\prime}=L$. If 2 is ramified in $K$ and $\operatorname{dim} V>2$, then $\operatorname{dim} V \equiv 0(\bmod 4)$.

(3) If $K$ is quaternionic, then $\operatorname{dim} V \equiv 0(\bmod 4)$. If $\operatorname{dim} V \equiv 4(\bmod 8)$, then $S$ is empty and $L_{\varphi}^{\prime}=L$.

Proof. (1) Since $R=\mathbf{Z}$ is unramified, $L^{\prime}=L$ is unimodular. The map $v \mapsto \varphi(v, v)(\bmod 2)$ gives a $G$-invariant linear form on $L / 2 L$. By our irreducibility hypothesis, this must be zero when $\operatorname{dim} V>1$, so $L$ is even. 
The fact that $\operatorname{rank} L \equiv 0(\bmod 8)$ for even unimodular $L$ is well known [S2, Chapter V, §2; MH, Theorem 5.1].

(2) Clearly $\operatorname{dim} V$ is divisible by $\operatorname{dim} K=2$. If $\operatorname{dim}_{K} V$ is odd, then $\operatorname{dim} V_{\mathfrak{p}}$ is odd for all ramified $\mathfrak{p}$. Hence $V_{\mathfrak{p}}$ is not symplectic, and $\mathfrak{p} \notin S$ by Proposition 7.2 (2). (The same argument proves (3).) If 2 is ramified in $K$ and $\operatorname{dim} V>2$, the form $\varphi_{\mathrm{p}}$ is strictly alternating in all cases, so $\operatorname{dim} V \equiv 0$ $(\bmod 4)$.

Corollary 7.5. If $\operatorname{dim} V \equiv 1(\bmod 2)$ then $K=\mathbf{Q}$ and $\operatorname{dim} V=1$. If $\operatorname{dim} V \equiv$ $2(\bmod 4)$ then $K=\mathbf{Q}(i)$ and $\operatorname{dim} V=2$, or $K=\mathbf{Q}(\sqrt{-N})$ is imaginary quadratic with odd discriminant $N \equiv 3(\bmod 4)$.

\section{The DUAL LatTice}

We henceforth assume $\operatorname{dim} V>1$, to exclude trivial cases. Let $\langle\rangle=,\operatorname{Tr} \varphi$ be the canonical positive-definite bilinear form on an $R G$-lattice $L$ in $V$. We define the (Euclidean) dual lattice by

$$
L^{*}=\{v \in V:\langle v, L\rangle \in \mathbf{Z}\} .
$$

This is equal to $L^{\prime}$ when $R=\mathbf{Z}$, but is a larger $R G$-lattice in $V$ when $R$ has ramified primes.

Recall that the inverse different $\mathscr{D}^{-1}$ of $R$ is the largest fractional ideal on which the trace takes integral values:

$$
\mathscr{D}^{-1}=\{\alpha \in K: \operatorname{Tr}(\alpha R) \in \mathbf{Z}\} .
$$

The integral ideal $\mathscr{D}$ is divisible precisely by the ramified primes $\mathfrak{p}$ of $R$; we have $\operatorname{ord}_{\mathfrak{p}}(\mathscr{D})=1$ except when $K$ is imaginary quadratic and $\mathfrak{p}^{2}=2 R$, when $\operatorname{ord}_{\mathfrak{p}}(\mathscr{D})=2$ or 3 . We say $R$ is tamely ramified when $\mathscr{D}$ is squarefree.

Proposition 8.3. We have $L^{*}=\mathscr{D}^{-1} L^{\prime}$. The form $\langle$,$\rangle is integral and even on$ $L$.

Proof. Since $L$ is an $R$-module, we have $\operatorname{Tr} \varphi(v, L) \in \mathbf{Z}$ if and only if $\varphi(v, L) \in \mathscr{D}^{-1}$. Hence $L^{*}=\mathscr{D}^{-1} L^{\prime}$.

By Proposition 5.2 we have $L^{\prime}=\left(\prod_{S} \mathfrak{p}\right) L$, hence $L^{*}=\left(\mathscr{D} / \prod_{S} \mathfrak{p}\right)^{-1} L$. Since the ideal $\mathscr{D} / \prod_{S} \mathfrak{p}$ is integral, $L^{*}$ contains $L$ and $\langle$,$\rangle is integral on L$.

To show $\langle$,$\rangle is even, we must show that \langle v, v\rangle \in 2 Z$ for all $v \in L$. If $K=\mathbf{Q}$ this was proved in Proposition 7.4 (1) (under the assumption that $\operatorname{dim} V>1)$. If $K \neq \mathbf{Q}$ we have $\langle v, v\rangle=2 \varphi(v, v)$, so we must show $\varphi(v, v) \in$ $\mathbf{Z}$. Since $\mathbf{Z}=\mathbf{Q} \cap \prod \mathbf{Z}_{p}$, it suffices to show that $\varphi(v, v) \in \mathbf{Z}_{p}$ for all primes $p$. This is clear when $p$ does not ramify in $R$, as $\varphi$ on $L_{p}$ takes values in $R_{p}$. If $p$ is ramified, let $\pi$ be a uniformizing parameter in $R_{p}$. Then $\pi \varphi$ on $L_{p}$ takes values in $R_{p}$. Hence $\varphi(v, v)$ lies in $\pi^{-1} R_{p} \cap \mathbf{Q}_{p}=\mathbf{Z}_{p}$.

If $L=\bigoplus_{i=1}^{n} \mathrm{Z} e_{i}$, we define the positive integer

$$
\operatorname{det} L=\operatorname{det}\left(\left\langle e_{i}, e_{j}\right\rangle\right) \text {. }
$$


This is independent of the basis chosen, and we have $\operatorname{det} L=\left(L^{*}: L\right)$. If $\operatorname{det} L=1$, or equivalently, $L^{*}=L$, we say $L$ is unimodular.

Note 8.5. Let $A$ be a finite abelian group $L^{*} / L$. Then $\langle$,$\rangle takes rational$ values on $L^{*}$, and induces a pairing $A \times A \rightarrow \mathbf{Q} / \mathbf{Z}$ which identifies $A$ with its Pontryagin dual. The unimodular $M$ with $L \subseteq M \subseteq L^{*}$ correspond bijectively to subgroups $B$ of $A$ which are their own annihilators $\left(B=B^{\perp}\right)$ under this pairing.

Proposition 8.6. If $R$ is tamely ramified, we have an isomorphism of finite $R[G]$ modules:

$$
L^{*} / L \simeq \bigoplus_{\mathfrak{p} \notin S} V_{\mathfrak{p}}
$$

The pairing $\langle$,$\rangle on L^{*} / L$ is the sum of the symmetric bilinear forms $\langle,\rangle_{p}=$ $\operatorname{Tr} \varphi_{\mathfrak{p}}$ on $V_{p}$ defined in Proposition 7.1 (1).

Proof. The hypothesis of tame ramification implies that $L^{*}=\left(\mathscr{D} / \prod_{\mathfrak{p} \in S} \mathfrak{p}\right)^{-1} L$ $=\left(\prod_{\mathfrak{p} \in S} \mathfrak{p}\right)^{-1} L$, so $L^{*} / L \simeq \bigoplus_{\mathfrak{p} \notin S} \mathfrak{p}^{-1} L / L \simeq \bigoplus_{\mathfrak{p} \notin S} V_{\mathfrak{p}}$. The identification of the bilinear forms follows from the fact that $\varphi_{p}$ is the reduction of $\varphi$.

\section{Theta SERIES}

In this section, we assume $R$ is tamely ramified, for simplicity. Let $\mathfrak{n}$ be the integral ideal $\prod_{\mathfrak{p} \notin S} \mathfrak{p}$ of $R$, and let $N$ be the positive integral generator of the ideal $\mathfrak{n} \cdot \overline{\mathfrak{n}}$.

We have seen in $\S 7$ that the assumption $\operatorname{dim} V>1$ implies $\operatorname{dim} V \equiv 0$ $(\bmod 2)$. Write $\operatorname{dim} V=2 k$. If $k$ is even, let $\varepsilon$ be the trivial character of the group $(\mathbf{Z} / N \mathbf{Z})^{*}$. If $k$ is odd, then by Proposition 7.1 (2), we have $K$ imaginary quadratic of discriminant $=-N$. Let $\varepsilon$ be the quadratic character of $(\mathbf{Z} / N \mathbf{Z})^{*}$ associated to the extension $K: \varepsilon(l)=+1$ iff the prime $l$ splits in $K$.

If $L$ is an $R G$-lattice in $V$, we define its theta series by

$$
\theta_{L}=\sum_{v \in L} e^{\pi i\langle v, v\rangle \tau}=\sum_{n \geq 0} \operatorname{Card}\left(L_{2 n}\right) q^{n},
$$

where $\tau$ is in the upper half-plane, $q=e^{2 \pi i \tau}$, and $L_{2 n}=\{v \in L:\langle v, v\rangle=2 n\}$. This series is easily shown to be convergent, and defines a holomorphic function in $\tau$.

Proposition 9.2. The function $\theta_{L}(\tau)$ is a holomorphic modular form of weight $k$ and character $\varepsilon$ for the group $\Gamma_{0}(N)$.

If $w_{N}$ is the Fricke involution which normalizes $\Gamma_{0}(N)$, we have the formula: $\theta_{L} \mid w_{N}=(-i)^{k} \theta_{\mathrm{n} L}$.

Proof. This follows from the results in $\S 8$ and the formulae in [Sch]. We omit the details. 
We note that the level, weight, and character of $\theta_{L}$ depend only on the representation $V$ of $G$, and not on the $R G$-lattice $L$ chosen. Hence the sum

$$
\theta=\sum_{L} \frac{1}{w_{L}} \cdot \theta_{L}
$$

over the $\mathbf{Z}[G]$-lattices $L$ (up to isomorphism) in $V$, with $\operatorname{End}_{G} L$ a maximal order in $K$ and $w_{L}=\operatorname{Card}\left(\operatorname{Aut}_{G} L\right)$, is a modular form of the same type.

More generally, for $n \geq 0$ let $\gamma_{2 n}(L)$ denote the permutation representation of $G$ on the finite set $L_{2 n}$. Then the Thompson series

$$
\left\{\begin{array}{l}
\Gamma_{L}=\sum_{n \geq 0} \gamma_{2 n}(L) \cdot q^{n}, \\
\Gamma=\sum_{L} \frac{1}{w_{L}} \cdot \Gamma_{L}=\sum_{n \geq 0} \gamma_{2 n} \cdot q^{n},
\end{array}\right.
$$

with coefficients in the rational representation ring of $G$, are interesting invariants of $L$ and $V$ respectively. We note that $w_{L}=\operatorname{Card}\left(R^{*}\right)=w_{R}$, where End $_{G} L=R$. Hence

$$
\gamma_{0}=\sum_{R} h_{R} / w_{R},
$$

where the sum is taken over the conjugacy classes of maximal orders $R$ in $K$ and $h_{R}=\operatorname{Card}(C l(R))$. In particular, $\gamma_{0}$ is a multiple of the trivial representation that depends only on the field $K=\operatorname{End}_{G}(V)$. The higher coefficients $\gamma_{2 n}$ are much more difficult to determine. An interesting invariant of $V$ is the minimal value of $n>0$ such that $\gamma_{2 n} \neq 0$.

\section{Examples when $K=\mathbf{Q}$ (cf. [Th1, Th2] )}

Unfortunately, this section is rather short. We have seen that if $V$ is a globally irreducible representation of $G$ with $\operatorname{dim} V>1$ and $K=\operatorname{End}_{G}(V)=$ $\mathbf{Q}$, then $\operatorname{dim} V \equiv 0(\bmod 8)$ as $L$ is even and unimodular. There are only three examples known where $L$ is indecomposable! They are listed in the following table, using the notation of the ATLAS [C]; in each case the representation $V$ of $G$ is uniquely determined by its dimension.

\begin{tabular}{|c|c|}
\hline$G$ & $\operatorname{dim} V$ \\
\hline$W\left(E_{8}\right)=2 . O_{8}^{+}(2) .2$ & 8 \\
$.0=2 . C o_{1}$ & 24 \\
$2 . T h$ & 248 \\
\hline
\end{tabular}

The stable lattices are the $E_{8}$-root lattice, the Leech lattice, and the ThompsonSmith lattice, respectively. 


\section{EXAMPLES WHEN $K=\mathbf{Q}(\sqrt{-p})$ AND $G=\operatorname{PSL}_{2}(p)$}

In this section, $p$ is a prime with $p \equiv 3(\bmod 4)$ and $K$ is the imaginary quadratic field $\mathbf{Q}(\pi)$ with $\pi^{2}=-p$. The unique maximal order $R$ is the ring $\mathbf{Z} \oplus \mathbf{Z}((1+\pi) / 2)$ and the unique ramified ideal is $\mathfrak{p}=\pi R$. The class group $C L(R)$ has odd order $h$; in the quadratic case $C l(R) / 2 C l(R)$ has order $2^{t-1}$ where $t$ is the number of ramified primes in $K$. For each globally irreducible $V$ with $\operatorname{End}_{G}(V)=K$ we obtain exactly $h \quad \mathrm{Z}[G]$-Euclidean lattices (or $R[G]$ Hermitian lattices) $L$ up to isomorphism in $V$.

In general, the lattices $L$ constructed form a principal homogeneous space for the class group $C l(R)$, but when a larger group acts on $V$ we can often fix a basepoint and index the lattices naturally by ideal classes. For example, if $G^{\prime}=G \rtimes\langle\tau\rangle$ acts on $V$ as in Lemma 5.5, there is a unique isomorphism class $L$ stable under $\tau$, as if $\mathfrak{a}$ is an ideal with nontrivial class, then $\mathfrak{a}$ is not equivalent to $\overline{\mathfrak{a}}$ in the class group (there are no nontrivial classes of order 2 ).

If $L$ is an $R G$-lattice in $V$, there are only two possibilities for the dual lattice, by Proposition 8.6:

$$
L^{*}= \begin{cases}L, & \operatorname{det} L=1, \quad V_{\mathrm{p}} \text { symplectic, } \\ \pi^{-1} L, & \operatorname{det} L=p^{k}, \quad V_{\mathrm{p}} \text { orthogonal. }\end{cases}
$$

Here $k=\frac{1}{2} \operatorname{dim} V=\operatorname{dim}_{K} V=\operatorname{dim} V_{\mathrm{p}}$. In the first case, $\theta_{L}$ is a modular form of weight $k$ on $\operatorname{SL}_{2}(Z)$; in the second $\theta_{L}$ is modular of weight $k$ on $\Gamma_{0}(p)$, with quadratic character $\varepsilon(d)=\left(\frac{d}{p}\right)^{k}$, and by Proposition 9.2 we have $\theta_{L} \mid w_{p}=(-i)^{k} \theta_{L}$.

A sequence of examples with $k=(p-1) / 2 \equiv 1(\bmod 2)$ was constructed by Hecke $[\mathrm{H}, \mathrm{A}]$.

Proposition 11.2. Let $G=\mathrm{PSL}_{2}(p)=\mathrm{SL}_{2}(p) /\langle \pm 1\rangle$ of order $\frac{1}{2} p\left(p^{2}-1\right)$. There is a unique irreducible representation $V$ of $G$ of dimension $(p-1)$ over $\mathbf{Q}$ with End $_{G}(V)=K$. The representation $V$ is globally irreducible, and the reduction $V_{\mathrm{p}}$ is orthogonal.

Proof. Let $\psi$ be the complex character of a $\frac{1}{2}$-discrete series representation of $G$, which has dimension $(p-1) / 2$ and $\mathbf{Q}(\psi)=K$ [Hu]. At an unramified prime of $R$, the reduction of $\psi$ is an irreducible Brauer character: in fact, its restriction to a Borel subgroup $B=\left\{\left(\begin{array}{cc}a & b \\ 0 & a^{-1}\end{array}\right)\right\}$ of $\mathrm{SL}_{2}(p)$ remains absolutely irreducible! This follows from the fact that the restriction of $\psi$ to the unipotent subgroup $N=\left\{\left(\begin{array}{ll}1 & b \\ 0 & 1\end{array}\right)\right\}$ contains $(p-1) / 2$ distinct characters, which are permuted transitively by $B$. Since these characters remain distinct in all characteristics $\neq p, \psi \equiv \rho$ is absolutely irreducible.

The reduced representation at $\mathfrak{p}$ is isomorphic to $\operatorname{Sym}^{(p-3) / 2} U_{2}$, where $U_{2}$ is the standard two-dimensional representation of $\mathrm{SL}_{2}(p)$ in characteristic $p$. This follows from [A, §4]; we will give another argument using hyperelliptic curves at the end of this section. This is an absolutely irreducible, orthogonal 
representation of $G$ over $\mathbf{F}_{p}$. Hence, by Proposition 4.2(2), $\chi=\psi+\bar{\psi}$ is the character of a globally irreducible representation $V$ of $G$.

To show $V$ is unique, we observe $V \otimes \mathbf{Q}=W \oplus \bar{W}$ where $W$ has character $\psi$. If $V^{\prime}$ were another such representation, $V^{\prime} \otimes \mathbf{C}=W^{\prime} \oplus \bar{W}^{\prime}$ and it suffices to show $W^{\prime}=W$ or $\bar{W}$ (two rational representations that are isomorphic over $\mathbf{C}$ are isomorphic over $\mathbf{Q}$ ). But $W$ and $\bar{W}$ are the only irreducible representations of dimension $(p-1) / 2$ for $G$ (at least when $p \neq 3$; when $p=3$ they are the only one-dimensional representations with $W \neq \bar{W}$ ).

Lemma 11.3. The group $G^{\prime}=\operatorname{PGL}_{2}(p)=G \rtimes\langle\tau\rangle$ with $\tau=\left(\begin{array}{cc}-1 & 0 \\ 0 & 1\end{array}\right)$ acts Qlinearly on $V$, and the involution $\tau$ acts $K$-antilinearly.

Proof. Indeed, $V$ is the representation space for the discrete series representation of $\mathrm{GL}_{2}(p)$, corresponding to a quartic character of the nonsplit torus. Since this is absolutely irreducible, $\operatorname{End}_{G^{\prime}}(V)=\mathbf{Q}$ and $\tau$ acts antilinearly on $\operatorname{End}_{G}(V)=K$.

From Proposition 11.2 and Lemma 11.3 we see that there are exactly $h$ $\mathbf{Z}[G]$-lattices $L$ up to isomorphism in $V$, indexed canonically by elements of $C l(R)$. We let $L_{1}$ be the class fixed by $\tau$, and $L_{a}=\mathfrak{a} L_{1}$. Each lattice $L$ has invariants:

$$
\left\{\begin{array}{l}
\operatorname{rank} L=p-1, \\
L^{*}=\pi^{-1} L, \\
\operatorname{det} L=(p)^{(p-1) / 2}
\end{array}\right.
$$

When $p=3, L$ is the $A_{2}$-root lattice; the representation $V$ of $G$ factors through the quotient $\mu_{3}$. When $p=7, L$ is the lattice described in $\S 6$; the form $\varphi$ is given by (6.2).

A cyclotomic model for the lattices $L_{\mathfrak{a}}$ in $V$ is due to Adler [A, §5]. Let $\zeta_{p}$ be a $p$ th root of unity in $\mathbf{C}$, and recall that $K=\mathbf{Q}(\sqrt{-p})$ is a subfield of the cyclotomic field $\mathbf{Q}\left(\zeta_{p}\right)$. We may realize the representation $V$ of $G$ on $\mathbf{Q}\left(\zeta_{p}\right)$ in a way that the unipotent element $\left(\begin{array}{ll}1 & 1 \\ 0 & 1\end{array}\right)$ acts by multiplication by $\zeta_{p}$, and $\left(\begin{array}{cc}a^{-1} & 0 \\ 0 & a\end{array}\right)$ acts via the element in the Galois group taking $\zeta_{p}$ to $\zeta_{p}^{a^{2}}$. If $\mathfrak{a}$ is an ideal of $R$, we let $i(\mathfrak{a})$ be the extended ideal $\mathfrak{a Z}\left[\zeta_{p}\right]$. Then

$$
L_{\mathfrak{a}}=\mathfrak{i}(\mathfrak{a}) \cdot\left(1-\zeta_{p}\right)^{-((p-3) / 4)}
$$

with $G$-invariant Hermitian form:

$$
\varphi(v, w)=\frac{1}{\mathbf{N a}} \operatorname{Trace}_{\mathbf{Q}\left(\zeta_{p}\right) / K}(v \bar{w}) .
$$

In particular, taking $\mathfrak{a}=(\pi)$, so $\mathfrak{i}(\mathfrak{a})=\left(1-\zeta_{p}\right)^{(p-1) / 2} \mathbf{Z}\left[\zeta_{p}\right]$, we see that $L_{1}$ is isomorphic to the Craig lattice $A_{p-1}^{(m)}$ with $m=(p+1) / 4$ [CS, p. 223].

Another model for the lattices $L_{a}$ was shown to me by Elkies; for the rest of this section, all varieties and morphisms between varieties are understood to be defined over the prime field $\mathbf{F}_{p}$. Let $X$ be the hyperelliptic curve, of genus 
$g=(p-1) / 2$, defined by the equation $y^{2}=x^{p}-x$, and let $J_{X}$ be the Jacobian of $X$. The group $G=\operatorname{PSL}_{2}(p)$ acts on $X$ by

$$
\left(\begin{array}{ll}
a & b \\
c & d
\end{array}\right)(x, y)=\left(\frac{a x+b}{c x+d}, \frac{y}{(c x+d)^{(p+1) / 2}}\right) .
$$

The group $\operatorname{Aut}(X)$ is isomorphic to the direct product of $G$ with the hyperelliptic involution $(x, y) \mapsto(x,-y)$, which acts as -1 on $J_{X}$. Let $E$ be the elliptic curve with equation $v^{2}=u^{3}-u$.

Lemma 11.8. The Frobenius endomorphism $F_{E}$ of $E$ satisfies $F_{E}^{2}=-p$ in $\operatorname{End}(E)$. We have $\operatorname{End}(E) \simeq R$, and for each ideal (class) a of $R$ there is a unique elliptic curve $E_{\mathfrak{a}}$ with $\operatorname{End}\left(E_{\mathfrak{a}}\right)=R$ and $\operatorname{Hom}\left(E, E_{\mathfrak{a}}\right) \simeq \mathfrak{a}$ as an $R$-module.

Proof. $E$ is the reduction of the curve $V^{2}=U^{3}-U$ over $\mathbf{Q}$, which has multiplication by $\mathbf{Z}[i]$ over $\mathbf{C}$. Hence the reduction is supersingular $(\bmod p)$, as $p \equiv 3$ (4) is inert in $\mathrm{Z}[i][\mathrm{S} 1, \S 2]$. For $p>3$ this implies $E$ has exactly $p+1$ points over $\mathbf{F}_{p}$ and hence that $F_{E}^{2}=-p$; for $p=3$ we can check this directly. Consequently, $\mathbf{Z}[\pi] \subset \operatorname{End}(E)$ with $\pi^{2}=-p$. To show $\operatorname{End}(E)=R$ we must show that $1+\pi$ is divisible by 2 in $\operatorname{End}(E)$, or equivalently, that all 2-torsion points on $E$ are rational over $\mathbf{F}_{p}$. This is clear, as the polynomial $u^{3}-u=u(u-1)(u+1)$ factors completely. The existence and uniqueness of the curve $E_{\mathfrak{a}}$ follows from Serre's theory of complex multiplication (cf. [S1, §2] where $E_{\mathfrak{a}}$ is written as $\mathfrak{a} * E$ ).

We now define the Mordell-Weil lattice:

$$
\begin{aligned}
M_{\mathfrak{a}} & =\operatorname{Mor}\left(X, E_{\mathrm{a}}\right) / \text { translations } \\
& =\operatorname{Hom}\left(J_{X}, E_{\mathrm{a}}\right),
\end{aligned}
$$

where Hom means homomorphisms of abelian varieties over $\mathbf{F}_{p}$. This is the group of points, modulo torsion, on the elliptic curve $E_{\mathrm{a}}$ over the global function field of $X$. It is clearly an $R=\operatorname{End}\left(E_{\mathrm{a}}\right)$ and $G \subseteq \operatorname{Aut}(X)$ module, and has the invariant Hermitian form

$$
\varphi(v, w)=v \circ^{t} w \in R=\operatorname{End}\left(E_{\mathfrak{a}}\right),
$$

where ${ }^{t} w: E_{\mathfrak{a}} \rightarrow J_{X}$ is the dual homomorphism, and $E_{\mathfrak{a}}$ and $J_{X}$ are identified with their dual varieties via the standard principal polarizations.

Proposition 11.11. The $R G$-Hermitian lattice $M_{\mathfrak{a}}$ is isomorphic to $L_{\mathfrak{a}} \subseteq V$.

Proof. We first consider the representation of $G$ on the rational vector space $V^{\prime}=M_{\mathfrak{a}} \otimes \mathbf{Q}$, and claim that $V^{\prime} \simeq V$. Indeed, $\left(V^{\prime}\right)^{G}=0$ and $\operatorname{End}_{G}\left(V^{\prime}\right)$ contains $K$, so it suffices to show $\operatorname{dim} V^{\prime}=p-1$ (by the unicity of $V$ ). This follows from Tate's theorem [Tal] on isogenies of abelian varieties over fields, as one has $F_{J_{X}}^{2}=-p$ in $\operatorname{End}\left(J_{X}\right)$ and $F_{E}^{2}=-p$ in $\operatorname{End}(E)$ by Lemma 11.8 . 
(Actually, one does not need the full power of Tate's theorem in this case, as the calculation of Frobenius elements easily shows that $\operatorname{dim} V^{\prime} \leq p-1=2 g$. To show equality, it suffices to prove that $V^{\prime} \neq 0$. But $X$ is isogenous to the curve $X^{*}$ with equation $y^{2}=x^{p+1}-1$ and $E$ is isogenous to the curve $E^{*}$ with equation $v^{2}=u^{4}-1$, and there is an obvious map $\pi(x, y)=\left(x^{(p+1) / 4}, y\right)$ from $X^{*}$ to $E^{*}$. Hence there is a less obvious map (of degree $\left.(p+1) / 4\right)$ from $X$ to $E$.)

Since $M_{\mathrm{a}}$ is an $R G$-lattice in $V^{\prime} \simeq V$, it is isomorphic to one of the lattices $L_{\mathrm{b}}$. To show $M_{\mathrm{a}} \simeq L_{\mathrm{a}}$ it suffices to show $M_{1} \simeq L_{1}$; indeed, from the definition of the curve $E_{\mathfrak{a}}$ we find $M_{\mathfrak{a}} \simeq \mathfrak{a} M_{1}$. Since $L_{1}$ is the unique isomorphism class of $R G$-lattices in $V$ fixed by the involution $\tau$, it suffices to give an action of $\tau$ on $M_{1}$. Let $i$ be a 4th root of unity in $\mathbf{F}_{p^{2}}$ and define the automorphisms $i_{X}(x, y)=(-x, i y), i_{E}(u, v)=(-u, i v)$ of order 4 of $X$ and $E$ over $\mathbf{F}_{p^{2}}$. Then $\tau(m)=i_{E} \circ m \circ i_{X}$ gives the desired $R$-antilinear involution of $M_{1}$.

Corollary 11.12 (Elkies). For all $v \neq 0$ in $L_{a}$, we have $\varphi(v, v) \geq(p+1) / 4$.

Proof. We use the model $M_{\mathfrak{a}}=\operatorname{Mor}\left(X, E_{\mathfrak{a}}\right) /$ translations, where $v \neq 0$ corresponds to a covering $\pi_{v}: X \rightarrow E_{\mathrm{a}}$ and $\varphi(v, v)=\operatorname{deg} \pi_{v}$. Since $X$ has $(p+1)$ points over $\mathbf{F}_{p}$, all of which are Weierstrass points, their image (suitably translated to contain the origin) lies in the two-torsion subgroup of $E_{\mathrm{a}}$, which has order 4 . Hence $\operatorname{deg} \pi_{v} \geq(p+1) / 4$.

Note 11.13. The lattice $L_{1}$ has at least $p(p-1)$ vectors $v$ with $\langle v, v\rangle=$ $2 \varphi(v, v)=(p+1) / 2$, so the abstract estimate in Corollary 11.12 is best possible. These maps come from the obvious covering of $E^{*}$ by $X^{*}$, discussed in the proof of Proposition 11.11, and form two orbits under the action of $G$ (each with stabilizer the normalizer of a nonsplit torus).

Note 11.14. The fact that the reduction $V_{\mathfrak{p}}$ of $V$ at the ramified prime $\mathfrak{p}$ of $K$ is isomorphic to the representation $\operatorname{Sym}^{(p-3) / 2} U_{2}$ can be deduced easily from Proposition 11.11. Indeed, $V_{p}$ is just the representation of $G$ on the holomorphic differentials on $X$; since these have as basis the differentials $\left\langle d y, x d y, x^{2} d y, \ldots, x^{(p-3) / 2} d y\right\rangle$, we obtain the representation $\operatorname{Sym}^{(p-3) / 2} U_{2}$ of $G$ as claimed.

\section{EXAMPLES WHEN $K=$ HAMILTON'S QUATERnions: THE BARNES-WALL LATTICES}

In this section $K$ is the division ring of Hamilton's quaternions, with the usual relations $i^{2}=j^{2}=-1$ and $i j=-j i=k$. The maximal orders in $K$ are all conjugate to the Hurwitz order $R=\mathbf{Z} i+\mathbf{Z} j+\mathbf{Z} k+\mathbf{Z}\left(\frac{1}{2}(1+i+j+k)\right)$.

The ring $R$ has class number 1 ; its unique ramified ideal $\mathfrak{p}$ is principal, generated by $\pi=(1+i)$. The unit group

$$
\left.R^{*}=\langle \pm 1, \pm i, \pm j, \pm k,( \pm 1 \pm i \pm j \pm k) / 2)\right\rangle
$$


has order 24 , with normal subgroup $H_{8}=\langle \pm 1, \pm i, \pm j, \pm k\rangle$ the quaternion group of order 8 .

For each globally irreducible representation $V$ of a finite group $G$ with $\operatorname{End}_{G}(V)=K$, we obtain, by the theory in $\S \S 1-5$, a unique $R G$-Hermitian lattice $L$ up to isomorphism. There are only two possibilities for the dual lattice, by Proposition 8.6:

$$
L^{*}= \begin{cases}L, & \operatorname{det} L=1, \quad V_{\mathrm{p}} \text { symplectic, } \\ \pi^{-1} L, & \operatorname{det} L=2^{k}, \quad V_{\mathrm{p}} \text { Hermitian } .\end{cases}
$$

Here $k=\frac{1}{2} \operatorname{dim} V=2 \operatorname{dim}_{K} V$ is even. In the first case, $\theta_{L}$ has weight $k$ on $\mathrm{SL}_{2}(\mathbf{Z})$; in the second, $\theta_{L}$ has weight $k$ on $\Gamma_{0}(2)$ and $\theta_{L} \mid w_{2}=(-1)^{k / 2} \theta_{L}$ by Proposition 9.2. A sequence of examples of globally irreducible $V$, with $k=2^{n}, n \geq 1$, is constructed as follows.

Let $\tilde{A}$ be the extra-special 2-group (denoted $2_{-}^{1+2 n}$ in the ATLAS [C]) which is a central extension of an elementary abelian 2-group $A \simeq(\mathrm{Z} / 2)^{2 n}$ by $\mathrm{Z} / 2$, corresponding to a nondegenerate quadratic form $Q$ on $A$ of Arf invariant 1. This makes sense, as $H^{2}(A, \mathrm{Z} / 2)=\operatorname{Sym}^{2}\left(A^{*}\right)$ is the space of quadratic forms on $A$; for an enlightening discussion of extraspecial 2-groups and their representation theory, see $[\mathrm{Q}, \S \S 2-4 ; \mathrm{Gr}]$. We have an exact sequence

$$
0 \rightarrow \mathbf{Z} / 2 \rightarrow \tilde{A} \underset{\pi}{\longrightarrow} A \rightarrow 0
$$

for any $a \in A$ we let $\tilde{a}$ be an element of $\tilde{A}$ with $\pi(\tilde{a})=a$. Then $Q(a)=\tilde{a}^{2}$ and the associated bilinear form $\langle a, b\rangle=Q(a+b)-Q(a)-Q(b)$ on $A$ is given by the commutator: $\langle a, b\rangle=\tilde{a} \tilde{b} \tilde{a}^{-1} \tilde{b}^{-1}$. Let $B$ be a maximal isotropic subspace of $A$, which has dimension $n-1$ by our hypothesis on $\operatorname{Arf}(Q)$, and write $B^{\perp}=B \oplus E$ where $\operatorname{dim} E=2$ and $\left.Q\right|_{E}=x^{2}+x y+y^{2}$. Then $\widetilde{E}$ is a quaternion group of order 8 in $\tilde{A}$. We identify $\widetilde{E}$ with $\langle \pm 1, \pm i, \pm j, \pm k\rangle$ in $R^{*}$ and write $\tilde{A}$ in Heisenberg form

$$
\begin{aligned}
& \tilde{A}=\widetilde{E} \times B \times B^{*}, \text { with } B^{*}=\operatorname{Hom}(B, \mathbf{Z} / 2) \text { and multiplication, } \\
& (\varepsilon, b, \lambda)\left(\varepsilon^{\prime}, b^{\prime}, \lambda^{\prime}\right)=\left((-1)^{\left\langle b^{\prime}, \lambda\right\rangle} \varepsilon \varepsilon^{\prime}, b+b^{\prime}, \lambda+\lambda^{\prime}\right) .
\end{aligned}
$$

Let $V$ be the Schrödinger representation of $\tilde{A}$, given by its right action on the left $K$-vector space of functions $f$ on $B^{*}$ with values in $K$ :

$$
f^{(\varepsilon, b, \lambda)}(\mu)=f(\mu-\lambda) \cdot(-1)^{\langle b, \mu-\lambda\rangle} \cdot \varepsilon .
$$

Then $\operatorname{dim}_{K}(V)=2^{n-1}$.

If we view $V$ as a representation of $\tilde{A}$ over $\mathbf{Q}$, then $\operatorname{dim} V=2^{n+1}$ and End $_{\widetilde{A}}(V)=K$. The character $\chi$ of $V$ is equal to $2 \psi$, where $\psi$ is the unique irreducible complex character of $\tilde{A}$ on which the center acts nontrivially. The reduction of $V(\bmod l)$ is irreducible over $R / l R=M_{2}(\mathbf{Z} / l \mathbf{Z})$ for all unramified primes $l \neq 2$ in $R$, as $\tilde{A}$ is a 2 -group. The reduction at $\mathfrak{p}=(1+i) R$ 
dividing 2 is not irreducible for the same reason: the only irreducible representation of a 2-group in characteristic 2 is the trivial representation.

Note 12.4. A stable lattice $L\left(B^{*}\right)$ for $\tilde{A}$ on $V$ is the $R$-module of functions on $B^{*}$ with values in $R$. By (12.3) we see that the action of $\tilde{A}$ on $L\left(B^{*}\right) / \mathfrak{p} L\left(B^{*}\right)$ factors through the regular representation of the quotient $B^{*}$.

The unicity of $V$ shows that it gives rise to a projective complex representation of dimension $2^{n}$ of the group $\operatorname{Aut}(\widetilde{A})$. We have an exact sequence

$$
0 \rightarrow A \rightarrow \operatorname{Aut}(\tilde{A}) \rightarrow O_{2 n}^{-}(2) \rightarrow 0,
$$

where $A=\tilde{A} /$ center $=\operatorname{Inn} \operatorname{Aut}(\tilde{A})$, and $O_{2 n}^{-}(2)$ is the orthogonal group of the quadratic space $(A, Q)$ [Gr]. Let $\Omega_{2 n}^{-}$be the subgroup of index 2 in $O_{2 n}^{-}(2)$ of elements with trivial Dickson determinant, and let $\mathrm{Aut}_{+}(\tilde{A})$ be the corresponding subgroup of $\operatorname{Aut}(\tilde{A})$.

When $n=1, \widetilde{A}$ is the quaternion group and $\operatorname{Aut}(\widetilde{A})$ is isomorphic to $S_{4}$. The group $\mathrm{Aut}_{+}(\tilde{A})$ is isomorphic to $A_{4}$, and has a double cover $G=\mathrm{SL}_{2}(3)$ which contains $\tilde{A}$ as a normal subgroup with quotient $\Omega_{2}^{-}(2)=\mu_{3}$. The projective representation of $\operatorname{Aut}(\tilde{A})$ lifts to a linear action of $G$ on $V=K$ with End $_{G}(V)=K$. When $n>2$ we have $H^{2}\left(\Omega_{2 n}^{-}(2), \mathbf{C}^{*}\right)=H^{1}\left(\Omega_{2 n}^{-}(2), \mathbf{C}^{*}\right)=1$ $[\mathrm{K}, \S 8.4]$; hence the projective representation of $\operatorname{Aut}(\widetilde{A})$ lifts uniquely to a linear representation of dimension $2^{n}$ over $\mathbf{C}$ of the double cover $G=2_{-}^{1+2 n} \cdot \Omega_{2 n}^{-}(2)$ of $\mathrm{Aut}_{+}(\tilde{A})$. The character of this representation of $G$ takes rational values, and is given by the formula:

$$
\psi(g)=\left\{\begin{array}{l} 
\pm 2^{\operatorname{dim} \operatorname{ker}(\bar{g}-1) / 2}, \\
0 .
\end{array}\right.
$$

Here $\bar{g}$ is the image of $g$ in $\Omega_{2 n}^{-}(2)$, and the value $\psi(g) \neq 0$ if and only if $g \equiv 1$ in $\operatorname{Aut}(\tilde{C})$ where $C=\operatorname{ker}(g-1)$. The hypothesis that $\bar{g} \in \Omega_{2 n}^{-}(2)$ insures that $\operatorname{dim} \operatorname{ker}(\bar{g}-1)$ is even, so $\mathbf{Q}(\psi)=\mathbf{Q}$. (The character of the linear representation on the full double cover of $\operatorname{Aut}(\widetilde{A})$ takes values in a quadratic extension of $\mathbf{Q}$ : for $n=1$ this is the two-dimensional representation of $\widetilde{S}_{4}$ with character in $\mathbf{Q}(\sqrt{-2})$, which was discussed in $\S 6$.) Hence $G$ acts on $V$, extending the action of $\tilde{A}$, and $\operatorname{End}_{G}(V)=K$.

Proposition 12.7. The representation $V$ of dimension $2^{n+1}$ of $G=2_{-}^{1+2 n} \cdot \Omega_{2 n}^{-}(2)$ is globally irreducible. Let $L$ be an $R G$-lattice in $V$ and $\mathfrak{p}=(1+i) R$ the ramified prime dividing 2 . The representation $V_{\mathfrak{p}}=L / \mathfrak{p} L$ of $G$ has dimension $2^{n-1}$ over $k_{\mathfrak{p}}=\mathbf{F}_{4}$, and the subgroup $\tilde{A}$ acts trivially on $V_{\mathfrak{p}}$. The quotient $\Omega_{2 n}^{-}(2)$ acts irreducibly on $V_{p}$ via the restriction of a semi-spinorial representation of the algebraic group $\operatorname{Spin}_{2 n}\left(\overline{\mathbf{F}}_{2}\right)$. The representation $V_{\mathfrak{p}}$ is Hermitian if $n \equiv 1$ $(\bmod 2)$, and alternating if $n \equiv 0(\bmod 2)$. 
Proof. Everything has been proved except the irreducibility of $V_{\mathrm{p}}$. Consider the semisimplification of the reduction of the character $\psi(\bmod 2)$. This has dimension $2^{n}$ over $\mathbf{F}_{2}$; since $\tilde{A}$ is a 2 -group it acts trivially, and we obtain a semisimple representation of the quotient $\Omega_{2 n}^{-}(2)$ with character

$$
\psi(\bar{g}) \equiv \begin{cases}1 & \text { if } \bar{g}-1 \text { acts invertibly }, \\ 0 & \text { otherwise }\end{cases}
$$

The irreducible modules of $\Omega_{2 n}^{-}(2)$ come via restriction of the irreducible representations of $\Omega_{2 n}^{-}\left(\mathbf{F}_{2}\right)=\operatorname{Spin}_{2 n}\left(\overline{\mathbf{F}}_{2}\right)$ with highest weights $\lambda$ satisfying: $\lambda=$ $\sum_{i=1}^{n} a_{i} \omega_{i}$ with $0 \leq a_{i} \leq 1$ and $\omega_{i}$ the fundamental weights [St1, Chapter 13]. Let $T$ be a maximal torus in $\Omega_{2 n}$, and let $\varepsilon_{1}, \ldots, \varepsilon_{n}$ be the standard characters of $T$. From the formula for the character $\psi$ on $\Omega_{2 n}^{-}(2)$, one concludes that the $2^{n}$ characters of $T$ which occur in the corresponding semisimple representation of $\Omega_{2 n}$ are precisely those of the form: $\frac{1}{2} \sum_{i=1}^{n} \pm \varepsilon_{i}$.

But these characters break up into exactly two orbits for the Weyl group $W=N(T) / T$, each of size $2^{n+1}$ corresponding to the semi-spinorial representations of $\Omega_{2 n}$ with highest weights $\omega_{n-1}$ and $\omega_{n}$ [B, §13.4]. When restricted to $\Omega_{2 n}^{-}(2)$ these give irreducible conjugate representations of dimension $2^{n-1}$ over $\mathbf{F}_{4}$, so we have the congruence $\psi \equiv \rho+\rho^{2}(\bmod 2)$ with $\rho$ absolutely irreducible and $\mathbf{F}_{2}(\rho)=\mathbf{F}_{4}$. Hence $V_{\mathfrak{p}}$ is irreducible, and $\tilde{A}$ acts trivially on it (the subspace $F_{\mathrm{p}}^{\tilde{A}}$, which is nontrivial, is $G$-stable).

When $n$ is odd the dual of one semi-spinorial representation is the other $\left(\omega_{n-1}^{*}=\omega_{n}\right)$ and when $n$ is even the semispin representations are self-dual $\left(\omega_{n-1}^{*}=\omega_{n-1}, \omega_{n}^{*}=\omega_{n}\right)[\mathbf{B}, \S 13.4]$. Since, when restricted to $\mathbf{F}_{4}$, the representations are conjugate, this gives the asserted duality of $V_{p}$.

Combining (12.5) with (12.1), we have constructed a unique Hermitian $R G$ lattice $L$ in $V$, with

$$
\left\{\begin{array}{l}
\operatorname{rank} L=2^{n+1}, \\
\operatorname{det} L= \begin{cases}1 & n \equiv 0(\bmod 2), \\
2^{2^{n}} & n \equiv 1(\bmod 2) .\end{cases}
\end{array}\right.
$$

When $n=1, L$ is the $D_{4}$-lattice, and when $n=2, L$ is the $E_{8}$-lattice.

Proposition 12.9. For all $n \geq 1$ the Euclidean lattice $L$ is isomorphic to the Barnes-Wall lattice $B W_{2^{n+1}}$.

Proof. The Barnes-Wall lattices $B W_{m}$ for $m=2^{k} \geq 4$ are defined in [CS, Chapter $5, \S 6.5$, Chapter $8, \S 8.2 \mathrm{f}]$; one has $B W_{4}=D_{4}$ and $B W_{8}=E_{8}$ so we may assume $n \geq 3$ in Proposition 12.9. Then $B W_{2^{n+1}}$ has automorphism group $2_{+}^{1+(2 n+2)} \Omega_{2 n+2}^{+}(2)[\mathrm{BE}$, Chapter II, $\S \S 4-7]$, which contains the subgroup

$$
2_{-}^{1+2} \Omega_{2}^{-}(2) \times{ }_{\langle \pm 1\rangle} 2_{-}^{1+2 n} \Omega_{2 n}^{-}(2)
$$


(central product). Since $2_{-}^{1+2} \Omega_{2}^{-}(2) \simeq R^{*}$ and $2_{-}^{1+2 n} \Omega_{2 n}^{-}(2)=G, B W_{2^{n+1}}$ has an $R G$ structure. Since the representation of $G$ on $B W \otimes \mathbf{Q}$ is isomorphic to $V$ (as the center of $\tilde{A}$ acts nontrivially), $B W \simeq L$ by unicity.

Corollary 12.10. For all $v \neq 0$ in $L$, we have $\langle v, v\rangle \geq 2^{[(n+1) / 2]}$. The number of vectors $v$ with $\langle v, v\rangle=2^{[(n+1) / 2]}$ is equal to $2^{n+2}\left(2^{n}+1\right)\left(2^{n-1}+1\right) \cdots(2+1)$.

Proof. These results are known for the lattice $B W_{2^{n+1}}$, by a consideration of the Reed-Muller codes [CS, p. 151]. For more discussion of the Barnes-Wall lattices and the Reed-Muller codes from a group-theoretic point of view, see [BE, VV].

Note 12.11. Elkies has realized $L$ as a sublattice, of finite index, in the MordellWeil lattice:

$$
\begin{aligned}
M & =\operatorname{Mor}_{\mathbf{F}_{q^{2}}}(X, E) / \text { translations } \\
& =\operatorname{Hom}_{\mathbf{F}_{q^{2}}}\left(J_{X}, E\right),
\end{aligned}
$$

where $X$ is the hyperelliptic curve $y^{2}+y=x^{q+1}$ with $q=2^{n}$ and $E$ is the supersingular elliptic curve $u^{2}+u=v^{3}$. L is generated by the coverings of $E$ obtained by dividing $X$ by a maximal elementary abelian 2-group in $\tilde{A} \subset \operatorname{Aut}_{\mathrm{F}_{q^{2}}}(X)$ which does not contain the center of $\widetilde{A}[\mathrm{VV}, 9.3]$. These Galois coverings have degree $2^{n-1}$.

Note 12.12. The lattice $L\left(B^{*}\right)$ discussed in 12.4 is not stable under the entire group $G$, but the lattice $\sum_{B^{*}} L\left(B^{*}\right)$ is $G$-stable.

\section{EXAMPLes: THE WeIl RePRESENTATION of $\operatorname{Sp}_{2 n}(q)$}

In this section, $p$ is an odd prime and $q=p^{f}$. Let $A$ be a nondegenerate symplectic space of dimension $2 n$ over the finite field $\mathbf{F}_{q}$, and let $G=\operatorname{Sp}(i)=$ $\operatorname{Sp}_{2 n}(q)$ be the symplectic group of $A$. Then the center of $G$ has order 2, and is generated by the scalar transformation $-1_{A}$. The group $G$ is a normal subgroup of the symplectic similitudes $C \operatorname{Sp}(A)$, and conjugation by this larger group gives an outer automorphism $\tau$ (of order 2) of $G$.

Fix a splitting $A=B \oplus B^{*}$, where $B$ and $B^{*}$ are maximal isotropic subspaces of dimension $n$. We identify $B^{*}$ with the dual of $B$ and, for $b \in B$ and $\lambda \in B^{*}$, write this pairing as $\langle b, \lambda\rangle$ in $\mathbf{F}_{q}$. The Heisenberg group

$$
\begin{aligned}
& \tilde{A}=\mathbf{F}_{q} \times B \times B^{*} \text { with multiplication, } \\
& (\varepsilon, b, \lambda) \cdot\left(\varepsilon^{\prime}, b^{\prime}, \lambda^{\prime}\right)=\left(\varepsilon+\varepsilon^{\prime}+\left\langle b^{\prime}, \lambda\right\rangle, b+b^{\prime}, \lambda+\lambda\right)
\end{aligned}
$$

defines a central extension of $A$ by $\mathbf{F}_{q}=\{(\varepsilon, 0,0)\}$ :

$$
0 \rightarrow \mathbf{F}_{q} \rightarrow \tilde{A} \underset{\pi}{\longrightarrow} A \rightarrow 0 .
$$

The commutator $\left[\tilde{a}, \tilde{a}^{\prime}\right]$ gives the symplectic form on $A$. 
Fix a nontrivial character $\psi: \mathbf{F}_{q}^{+} \rightarrow \mathbf{C}^{*}$. The Schrödinger representation $W=W(\psi)$ of $\tilde{A}$ (associated to $\psi$ ) is given by the following right action of $\tilde{A}$ on the $\mathbf{Q}\left(\zeta_{p}\right)$ vector space of functions on $B^{*}$ with values in $\mathbf{Q}\left(\zeta_{p}\right)=\mathbf{Q}(\psi)$ :

$$
f^{(\varepsilon, b, \lambda)}(\mu)=\psi(\varepsilon+\langle b, \mu-\lambda\rangle) \cdot f(\mu-\lambda) .
$$

It is the unique irreducible representation of $\tilde{A}$ over $\mathbf{Q}\left(\zeta_{p}\right)$ (or even over $\mathbf{C}$ ) where the center acts by the character $\psi$, and we have $\operatorname{dim} W=q^{n}$.

The unicity of $W$ shows that it gives rise to a projective representation of the group of automorphisms of $\tilde{A}$ which act trivially on the center. This group contains $G=\operatorname{Sp}(A)$, which is the subgroup centralizing the involution $-1_{A}(\varepsilon, \lambda, b)=(\varepsilon,-\lambda,-b)$. Hence $G$ acts projectively on $W$ over $\mathbf{Q}\left(\zeta_{p}\right)$. Since $H^{2}\left(G, C^{*}\right)=0$ (except in the case when $n=1$ and $q=3$ or $3^{2}$ ) $[\mathrm{K}, \S 8.4]$ this projective representation lifts to a linear representation; since $H^{1}\left(G, \mathbf{C}^{*}\right)=0$ (except in the one case when $n=1$ and $q=3$ ) the lifting to a linear representation is unique. (A unique lifting for $\mathrm{SL}_{2}\left(3^{2}\right)$ exists (cf. [Ge, Lemma 1.5]), and a lifting for $G=\mathrm{SL}_{2}(3)$ exists and is specified in [Ge, Theorem $2.4 a^{\prime \prime}$ ].) We denote the lifted representation of $G$ again by $W(\psi)$; is the Weil representation of $G$, of dimension $q^{n}$, associated to the character $\psi$.

The Siegel parabolic $P$ of $G$, which fixes the isotropic subspace $B$, acts on $W(\psi)$ as follows [Ge, (2.7)-(2.9)]:

$$
\left\{\begin{array}{l}
f^{\left(\begin{array}{cc}
{ }^{\alpha} \alpha_{0}^{-1} & 0 \\
0
\end{array}\right)(\lambda)=\left(\frac{\operatorname{det} \alpha}{q}\right) \cdot f(\alpha \lambda),} \\
f^{\left(\begin{array}{cc}
1 & \beta \\
0 & 1
\end{array}\right)(\lambda)=\psi\left(\frac{1}{2}\langle\beta(\lambda), \lambda\rangle\right) \cdot f(\lambda) .}
\end{array}\right.
$$

Here $\alpha \in \mathrm{GL}\left(B^{*}\right)$ and $\beta={ }^{t} \beta: B^{*} \rightarrow B$ is symmetric; the symbol $\left(\frac{x}{q}\right)$ denotes the Legendre symbol-the unique quadratic character of $\mathbf{F}_{q}^{*}$. We have the commutation law:

$$
\left(\begin{array}{cc}
{ }^{t} \alpha^{-1} & 0 \\
0 & \alpha
\end{array}\right)^{-1}\left(\begin{array}{ll}
1 & \beta \\
0 & 1
\end{array}\right)\left(\begin{array}{cc}
t^{2} & 0 \\
0 & \alpha
\end{array}\right)=\left(\begin{array}{cc}
1 & { }^{t} \alpha \beta \alpha \\
0 & 1
\end{array}\right) .
$$

The action of the matrix $\left(\begin{array}{cc}0 & \beta \\ -\beta^{-1} & 0\end{array}\right)$, where $\beta: B^{*} \underset{\sim}{\sim}$ is invertible is given by a Fourier transform, but we will not need this fact here.

The distinct nontrivial characters of $\mathbf{F}_{q}^{+}$all have the form $\psi_{x}(y)=\psi(x y)$ for $x \in \mathbf{F}_{q}^{*}$.

Lemma 13.4. (1) We have an isomorphism of G-modules $W(\psi) \simeq W\left(\psi_{x}\right)$ if and only if $x$ is a square in $\mathbf{F}_{q}^{*}$. If $x \in \mathbf{F}_{q}^{*}-\mathbf{F}_{q}^{*^{2}}$, the distinct G-modules $W(\psi)$ and $W\left(\psi_{x}\right)$ are conjugate via the outer automorphism $\tau$ of $G$.

(2) If $a \in \mathbf{F}_{p}^{*}$ we have an isomorphism of G-modules $W\left(\psi_{a}\right)=W(\psi)^{\sigma_{a}}$, where $\sigma_{a}$ is the automorphism of $\mathbf{Q}\left(\zeta_{p}\right)$ which maps $\zeta_{p}$ to $\zeta_{p}^{a}$. In particular, the dual representation $W(\psi)^{*} \simeq \overline{W(\psi)}$ is isomorphic to $W\left(\psi_{-1}\right)$. 
Proof. (1) is proved in [Ge, Theorem 2.4], and (2) follows from the fact that $\psi^{\sigma_{a}}=\psi_{a}$.

In particular, we see that the Weil representation $W$ is self-dual if and only if $q \equiv 1(\bmod 4)$, when $\left(\frac{-1}{q}\right)=+1$. In general, the tensor product representation $W \otimes W^{*}$ is independent of $\psi$, and is isomorphic to the natural representation of $G$ (of dimension $q^{2 n}$ ) on the $\mathbf{Q}\left(\zeta_{p}\right)$-valued functions on $A$ [Ge, Theorem 4.4].

Lemma 13.5. (1) The Weil representation $W=W(\psi)$ of $G$ is the direct sum of 2 absolutely irreducible representations $W^{+}$and $W^{-}$over $\mathbf{Q}\left(\zeta_{p}\right)$. We have $\operatorname{dim} W^{ \pm}=\left(q^{n} \pm 1\right) / 2$ and the center $-1_{A}$ of $G$ acts on $W^{ \pm}$via the scalar $\pm\left(\frac{-1}{q}\right)^{n}$.

(2) Let $\chi=\chi^{+}+\chi^{-}$be the decomposition of the character of $W$. If $q$ is a square, then $\mathbf{Q}(\chi)=\mathbf{Q}\left(\chi^{+}\right)=\mathbf{Q}\left(\chi^{-}\right)=\mathbf{Q}$. If $q=p^{f}$ with $f$ odd, then

$$
\mathbf{Q}(\chi)=\mathbf{Q}\left(\chi^{+}\right)=\mathbf{Q}\left(\chi^{-}\right)= \begin{cases}\mathbf{Q}(\sqrt{-p}) & p \equiv 3(\bmod 4) \\ \mathbf{Q}(\sqrt{p}) & p \equiv 1(\bmod 4)\end{cases}
$$

Proof. (1) We have $\langle\chi, \chi\rangle=\langle 1, \chi \cdot \bar{\chi}\rangle=2$, as $G$ has 2 orbits on $A$. Hence the eigenspaces for $-1_{A}$ in $W$ are absolutely irreducible: the even functions on $B^{*}$ give $W^{+}$of dimension $\left(q^{n}-1\right) / 2$. The action of $-1_{A}$ is given by (13.3), taking $\alpha=-1_{B}$.

(2) If $q$ is a square, then any $a$ in $\mathbf{F}_{p}^{*}$ is a square in $\mathbf{F}_{q}^{*}$. Hence, by Lemma 13.4 (2), $\chi$ is fixed by the automorphisms of $\mathbf{Q}\left(\zeta_{p}\right)$. Since $\chi^{+}$and $\chi^{-}$are not conjugate, they must also lie in $\mathbf{Q}$. If $q$ is not a square, the same argument shows that $\mathbf{Q}(\chi)$ is the unique quadratic field contained in $\mathbf{Q}\left(\zeta_{p}\right)$.

We now determine the local behavior of the characters $\chi^{ \pm}$at all places of the field $\mathbf{Q}(\chi)$.

Proposition 13.6. Let $\mathfrak{p}$ be a finite place of $\mathbf{Q}(\chi)$. (1) If $\mathfrak{p}$ does not divide $2 p$, then the characters $\chi^{ \pm}$both have absolutely irreducible reduction $(\bmod \mathfrak{p})$.

(2) If $\mathfrak{p}$ divides 2 , then $\chi^{-}$has absolutely irreducible reduction $(\bmod \mathfrak{p})$ and $\chi^{+} \equiv 1+\chi^{-}(\bmod \mathfrak{p})$.

(3) If $\mathfrak{p}$ is the unique prime dividing $p$, then the characters $\chi^{ \pm}$have absolutely irreducible reduction $(\bmod \mathfrak{p})$ if and only if $f=1$ (that is, if $q=p$ ). In this case, the reduction is symplectic when $\chi^{ \pm}\left(-1_{A}\right)=-\chi^{ \pm}\left(1_{A}\right)$, and orthogonal when $\chi^{ \pm}\left(-1_{A}\right)=\chi^{ \pm}\left(1_{A}\right)$.

(4) The character $\chi^{+}$is unobstructed at all real places of $\mathbf{Q}(\chi)$, and the character $\chi^{-}$is obstructed at all real places of $\mathbf{Q}(\chi)$.

Proof. From (13.3), one sees that the restriction of $\chi^{-}$to the Siegel parabolic $P$ is irreducible in all characteristics $\neq p$. Similarly, the restriction of $\chi^{+}$to $P$ is the direct sum of the line of functions supported on 0 in $B^{*}$, on which $P$ acts via the character $((\operatorname{det} \alpha) / q)$, with an irreducible representation. This decomposition is preserved by $G$ only when $l=2: W^{+}$has the smallest 
dimension of an irreducible representation with central character not equal to that of $W^{-}$[LS]. This proves (1) and (2).

(3) The irreducible representations of $G=\operatorname{Sp}_{2 n}(q)$ in characteristic $p$ all have the form

$$
U=U_{1} \otimes U_{2}^{p} \otimes \cdots \otimes U_{f}^{p^{f-1}},
$$

where $U_{i}$ are irreducible algebraic representations of $\operatorname{Sp}_{2 n}\left(\overline{\mathbf{F}}_{p}\right)$ restricted to $G$ [St2]. If $\omega_{1}, \ldots, \omega_{n}$ are the fundamental dominant weights for $\mathrm{Sp}_{2 n}$, then the highest weights $\lambda_{i}$ of the irreducible representations $U_{i}$ have the form $\lambda_{i}=\sum_{j=1}^{n} a_{i j} \omega_{j}$ with $0 \leq a_{i j} \leq p-1$ [St1]. The high, $\mathrm{t}$ weight of $U$ is then $\lambda_{1}+p \lambda_{2}+p^{2} \lambda_{3}+\cdots+p^{f-1} \lambda_{f}=\sum_{j=1}^{n} b_{j} \omega_{j}$ with $0 \leq b_{j} \leq q-1$.

From (13.3) we see that the weights occurring in the reduction of $W$ are the characters

$$
\begin{gathered}
\lambda=\sum a_{i} e_{i} \text { with }-\left(\frac{q-1}{2}\right) \leq a_{i} \leq\left(\frac{q-1}{2}\right), \\
e_{i}\left(\begin{array}{ccccc}
t_{1} & & & & 0 \\
& \ddots & & & \\
& & t_{n} & & \\
& 0 & & t_{1}^{-1} & \\
& & & \ddots & \\
& & & & t_{n}^{-1}
\end{array}\right)=t_{i} .
\end{gathered}
$$

Each weight occurs with multiplicity one in $W$, and occurs in $W^{+}$iff $\sum a_{i} \equiv$ $n((q-1) / 2)(\bmod 2)$. In particular,

$$
\lambda^{+}=\sum((q-1) / 2) e_{i}=((q-1) / 2) \omega_{n}
$$

is the highest dominant weight of $W^{+}$, and

$$
\lambda^{-}=\sum((q-1) / 2) e_{i}-e_{n}=\omega_{n-1}+((q-3) / 2) \omega_{n}
$$

is the highest dominant weight in $W^{-}[\mathrm{B}, \S 13.3]$.

Let $U_{+}$be the irreducible algebraic representation of $\operatorname{Sp}_{2 n}\left(\overline{\mathbf{F}}_{p}\right)$ with highest weight $((p-1) / 2) \omega_{n}$, and let $U_{-}$be the irreducible algebraic representation with highest weight $\omega_{n-1}+((p-3) / 2) \omega_{n}$. By the above remarks, the semisimplification of $W^{+}(\bmod p)$ contains the irreducible representation $U_{+} \otimes U_{+}^{p} \otimes U_{+}^{p^{2}} \otimes \cdots \otimes U_{+}^{p^{\prime-1}}$ and the semi-simplification of $W^{-}(\bmod p)$ contains $U_{-} \otimes U_{+}^{p} \otimes U_{+}^{p^{2}} \otimes \cdots \otimes U_{+}^{p^{f-1}}$.

But it is known that for $q=p, W^{+} \equiv U_{+}$, and $W^{-} \equiv U_{-}$are irreducible [ZS]. Hence $\operatorname{dim} U_{ \pm}=\left(\left(p^{n} \pm 1\right) / 2\right)$. By a dimension count, $W^{ \pm}$is not irreducible once $f>1$. The autoduality of $U_{ \pm}$is determined by the action of the center $-1_{A}$ of $G$ : the representation is symplectic if $-1_{A}$ acts nontrivially and orthogonal if $-1_{A}$ acts trivially [St 1, Lemmas 78, 79]. 
(4) The field $\mathbf{Q}(\chi)$ has real places iff $q \equiv 1(\bmod 4)$, so we assume this is the case. Then $-1_{A}$ acts as \pm 1 on $W^{ \pm}$. The space $\operatorname{Hom}_{G}\left(W, W^{*}\right)$ has dimension 2, and transpose is not a scalar on it: hence one of $W^{ \pm}$is obstructed and one is unobstructed over $\mathbf{R}$, by the Frobenius-Schur criterion [S3, Proposition 39]. But $W^{+}$is a representation of $P \operatorname{Sp}_{2 n}(q)$, which is unobstructed by [Gol, Go2]. An alternate argument is to reduce to the case of $\mathrm{SL}_{2}(q)$ using [Ge, Corollary 4.4].

Corollary 13.7. (1) If $q \equiv 3(\bmod 4)$, a representation $V^{ \pm}$with character $\chi^{ \pm}$ can be defined over the imaginary quadratic field $K=\mathbf{Q}(\sqrt{-p})$.

(2) If $q \equiv 1(\bmod 4)$ but $f$ is odd, a representation $V^{+}$with character $\chi^{+}$ can be defined over the real quadratic field $K=\mathbf{Q}(\sqrt{p})$. A representation $V^{-}$ with character $2 \chi^{-}$can be defined over $K$, and $\operatorname{End}_{K[G]} V^{-}$is the quaternion division algebra over $K$ ramified at $\{\infty, \infty\}$.

(3) If $q$ is a square, a representation $V^{+}$with character $\chi^{+}$can be defined over $\mathbf{Q}$. A representation $V^{-}$with character $2 \chi^{-}$can be defined over $\mathbf{Q}$, and End $_{G} V^{-}$is the quaternion division algebra over $\mathbf{Q}$ ramïfied at $\{\infty, p\}$.

Proof. We use the criterion in Lemma 4.1 to see that the character $\chi^{ \pm}$is unobstructed at all places of $\mathbf{Q}\left(\chi^{ \pm}\right)=\mathbf{Q}(\chi)$ not dividing $p$ and $\infty\left(\chi^{+}\right.$is unobstructed at 2 as the irreducible pieces in its decomposition have multiplicity one and the same field of definition as $\chi^{+}$).

When $q \equiv 3(\bmod 4)$ there is only one possible place $\mathfrak{p}=(\sqrt{-p})$ of $K$ where $\chi^{ \pm}$can be obstructed. But any division algebra over $K$ which splits outside $\mathfrak{p}$ is globally split, by the Hasse reciprocity law. Hence the characters $\chi^{ \pm}$are unobstructed.

When $q \equiv 1(\bmod 4)$ the representation $\chi^{+}$can only be obstructed at $\mathfrak{p}$, so by the above argument is unobstructed. The representation $\chi^{-}$is obstructed by a quaternion algebra, as $e_{\mathbf{R}}\left(\chi^{-}\right)=2$, and the ramification of this algebra can be determined from the number of real places or $\mathbf{Q}\left(\chi^{-}\right)$(it must ramify at an even number of places).

Proposition 13.8. (1) If $p \equiv 3(\bmod 4)$ there is a unique globally irreducible representation $V$ of $G=\operatorname{Sp}_{2 n}(p)$ of dimension $p^{n}-1$ over $\mathbf{Q}$ with $\operatorname{End}_{G}(V)=$ $\mathbf{Q}(\sqrt{-p})$. The reduction $V_{\mathfrak{p}}$ at the ramified prime $\mathfrak{p}=(\sqrt{-p})$ is $(-1)^{n-1}$. symmetric.

(2) If $p$ is odd, there are two globally irreducible representations $V$, of $G=$ $\operatorname{Sp}_{2 n}\left(p^{2}\right)$ of dimension $p^{2 n}-1$ over $\mathbf{Q}$, with $\operatorname{End}_{G}(V)=K$, the definite quaternion algebra ramified at $\{p, \infty\}$. These two representations are conjugate by the outer automorphism $\tau$ of $G$. The reduction $V_{p}$ at the ramified prime dividing $p$ is symplectic.

Proof. (1) $V$ is the rational representation of $G$ on the $\mathbf{Q}(\sqrt{-p})$ vector space $V^{-}$of Corollary 13.7. It is globally irreducible by Proposition 13.6. Its reduction is $(-1)^{n-1}$-symmetric, as $-1_{A}$ acts as $-\left(\frac{-1}{p}\right)^{n}=(-1)^{n-1}$ on $V$ and $V_{p}$. 
(2) $V$ is the rational representation of $G$ on $V^{-}$of Corollary 13.7. We muse check that the reduction $V_{\mathfrak{p}}$ is irreducible; in fact the argument in the proof of Proposition 13.6 (3) shows that we have an isomorphism of $k_{\mathrm{p}}$ with $\mathbf{F}_{p^{2}}$ such that $V_{\mathrm{p}} \simeq U_{-} \otimes U_{+}^{p}$. This representation is symplectic, as $U_{-}$and $U_{+}$are both self-dual, with opposite parity.

Combining Proposition 13.8 with our basic construction of Hermitian $R G$ lattices, we obtain the following:

Corollary 13.9. If $p \equiv 3(\bmod 4)$ there are, up to isomorphism, $h=h(-p)$ Hermitian $R=\mathbf{Z}+\mathbf{Z}((1+\sqrt{-p}) / 2)$ lattices $L$ in $V$ which are stable under $G=\operatorname{Sp}_{2 n}(p)$. These lattices have invariants

$$
\left\{\begin{array}{l}
\operatorname{rank} L=p^{n}-1, \\
\operatorname{det} L= \begin{cases}p^{\left(p^{n}-1\right) / 2} & n \text { odd }, \\
1 & n \text { even } .\end{cases}
\end{array}\right.
$$

When $n=1$ these are Hecke's lattices, constructed in $\S 11$. In general, they are indexed canonically by elements of the class group of $R$, as the larger group $G^{\prime}=G \rtimes\langle\tau\rangle$, where $\tau$ is the similitude with matrix $\left(\begin{array}{cc}-1_{n} & 0_{n} \\ 0_{n} & 1_{n}\end{array}\right)$, acts Q-linearly on $V$.

Corollary 13.10. Let $p$ be odd, let $R$ be a maximal order in the quaternion algebra $K$ ramified at $p$ and $\infty$ and let $G=\operatorname{Sp}_{2 n}\left(p^{2}\right)$. Then there are $h_{R}\left(=1\right.$ or 2) Euclidean $\mathbf{Z}[G]$-lattices $L$ up to isomorphism in $V$ with $\operatorname{End}_{G}(L)$ $=R$. These lattices have invariants:

$$
\left\{\begin{aligned}
\operatorname{rank} L & =p^{2 n}-1, \\
\operatorname{det} L & =1 .
\end{aligned}\right.
$$

We note that the sum over the conjugacy classes of maximal orders $R$ in $K$ of the weighted class numbers:

$$
\sum_{R} h_{R} / w_{R}=\frac{p-1}{24}
$$

by Eichler's mass formula [Ei] (cf. [V, Chapter V, §2]). Since $w_{R}=\operatorname{Card}\left(R^{*}\right)=$ 2 for all but possibly 2 maximal orders, we have constructed about $(p-1) / 12$ $\mathbf{Z}[G]$-lattices in $V$ with $\operatorname{End}_{G}(L)$ a maximal order in $K$.

The unimodular lattices constructed in 13.9 are among the unimodular lattices constructed in 13.10. Indeed, the restriction of the Weil representation of $\mathrm{Sp}_{4 n}(p)$ to the subgroup $\mathrm{Sp}_{2 n}\left(p^{2}\right)$ stabilizing an $\mathbf{F}_{p^{2}}$-linear structure on $A$, is the Weil representation of $\operatorname{Sp}_{2 n}\left(p^{2}\right)$. We obtain the unimodular lattices $L$ of rank $p^{2 n}-1$ which are associated to those maximal orders $R$ in the quaternion division algebra which contain the maximal order $\mathbf{Z}+\mathbf{Z}((1+\sqrt{-p}) / 2)$ of $\mathbf{Q}(\sqrt{-p})$. These special lattices have automorphisms by $\operatorname{Sp}_{4 n}(p)$ commuting with $\sqrt{-p}$; I do not know their automorphism groups over $\mathbf{Z}$. 
For example, take $n=1$ and $p=3$. The unique lattice constructed is the $E_{8}$-lattice, with automorphisms by $\mathrm{Sp}_{4}(3)$ commuting with $\sqrt{-3}$. When $p=7$ the unique lattice constructed has rank 48 , automorphisms by $\operatorname{Sp}_{4}(7)$ commuting with $\sqrt{-7}$, and shortest vector $v$ satisfying $\langle v, v\rangle=4$. A model for the lattices when $G=\mathrm{SL}_{2}\left(p^{2}\right)$ is due to Elkies. Let $X$ be the hyperelliptic curve with equation $y^{2}=x^{p^{2}}-x$ over $\mathbf{F}_{p^{2}}$, and let $E$ be a supersingular ellip tic curve with Frobenius $=-p$ and $\operatorname{End}(E)=R$. Then $L=\operatorname{Mor}_{\mathrm{F}_{p^{2}}}(X, E) /$ translations $=\operatorname{Hom}_{\mathbf{F}_{p^{2}}}\left(J_{X}, E\right)$ with Hermitian form $\varphi(v, w)=\frac{1}{p} v o^{t} w$. Arguing as in 11.11, we obtain the estimate $\langle v, v\rangle \geq(p+1) / 2$ for $v \neq 0$ in $L$, with equality holding for some $v \in L$ in the case when $p \equiv 3(\bmod 4)$ and $j(E)=1728$. The group $\operatorname{Sp}_{4}(p)$ acts on $L$ when $E$ can be defined over $\mathbf{F}_{p}$.

I know of no good model for the unimodular lattices with automorphisms by $\mathrm{Sp}_{2 n}\left(p^{2}\right)$ (or $\mathrm{Sp}_{4 n}(p)$ ) once $n>1$. In particular, $\mathrm{I}$ have no estimate for their shortest vectors.

\section{EXAMPLES: THE UNIPOTENT CUSPIDAL REPRESENTATION OF $\operatorname{PU}_{3}(q)$}

In this section, $p$ is an arbitrary prime number and $q=p^{f}$. We let $G$ be the finite group $\mathrm{PU}_{3}(q)$, the projective unitary group of a nondegenerate Hermitian form in 3-variables over $\mathbf{F}_{q^{2}}$. The group $G$ has order $=$ $\left(q^{3}+1\right)\left(q^{3}\right)\left(q^{2}-1\right)$ acts 2-transitively on the set of $q^{3}+1$ isotropic lines. It acts by algebraic automorphisms of the Fermat curve $X$ with equation $x^{q+1}+y^{q+1}+z^{q+1}=0$ in characteristic $p$; indeed this equation may be rewritten as $x \bar{x}+y \bar{y}+z \bar{z}=0$ over $\mathbf{F}_{q^{2}}$, where $\bar{\alpha}=\alpha^{q}$ is the nontrivial involution of $\mathbf{F}_{q^{2}}$ over $\mathbf{F}_{q}$.

We let $\psi$ denote the character of the irreducible cuspidal unipotent representation, of dimension $q(q-1)$, of $G$ over $\mathbf{C}$ [Ca, §13.7]. Then $\mathbf{Q}(\psi)=\mathbf{Q}$, and for all $l \neq p$ a model for the representation with character $\psi$ over $\mathbf{Q}_{l}$ is given by the action of $G$ on the étale cohomology $H^{1}\left(X, \mathbf{Q}_{l}\right)$ of the Fermat curve over an algebraically closed field of characteristic $p$ [HM]. It is known that $e_{\mathbf{R}}(\psi)=2$; this follows from the Frobenius-Schur criterion, as $G$ fixes the alternating form on $H^{1}(X)$ (cf. [L, Proposition 7.6]). Hence $e(\psi)=2$ and $\psi$ must be obstructed at $p$. We let $V$ be the irreducible representation of dimension $2 q(q-1)$ for $G$ over $\mathbf{Q}$ with character $2 \psi$. Then $\operatorname{End}_{G}(V)=K$, the quaternion division algebra over $\mathbf{Q}$ ramified at $p$ and $\infty$.

Proposition 14.1. The representation $V$ of $G=\mathrm{PU}_{3}(q)$ is irreducible over $\mathbf{R}$, and for all primes $l \neq p$ its reduction is irreducible over $k_{l}=M_{2}(\mathbf{Z} / l \mathbf{Z})$. If $\mathfrak{p}$ is the unique ramified prime in $K$, the reduction $V_{p}$ is irreducible over $k_{p}=F_{p^{2}}$ if and only if $q=p$ or $q=p^{2}$. When $q=p$ the representation $V_{p}$ is an absolutely irreducible Hermitian representation: when $q=p^{2}$ the representation $V_{\mathfrak{p}}$ is symplectic and $\operatorname{End}_{k_{\mathrm{p}}}\left(V_{\mathrm{p}}\right)=\mathbf{F}_{p^{4}}$. 
Proof. We have already seen that $V$ is irreducible over $\mathbf{R}$. It is irreducible $(\bmod l)$ by the results of $\S 4$, as $\psi \equiv \rho$ is an absolutely irreducible Brauer character for all primes $l \neq p$. This follows from the fact that $q(q-1)$ is the smallest dimension of an absolutely irreducible, nontrivial representation of $G$ in any characteristic $\neq p$ [LS]. One can prove it directly by restricting to a Borel subgroup fixing an isotropic line, as in Proposition 11.2.

When $l=p$, the representation with character $\psi$ over the Witt vectors $W_{q^{2}}$ of $\mathbf{F}_{q^{2}}$ is given by the action of $G$ on the crystalline cohomology group $H^{1}\left(X, W_{q^{2}}\right)$. The representation of $G$ with character $\psi(\bmod p)$ is therefore given by the action of automorphisms on the de Rham cohomology, $H_{d R}^{1}\left(X, \mathbf{F}_{q^{2}}\right)$ $=H^{1}\left(X, W_{q^{2}}\right) / p H^{1}\left(X, W_{q^{2}}\right)$. We have then an isomorphism of $G$-modules over $\mathbf{F}_{q^{2}}$ :

$$
V_{\mathfrak{p}} \otimes_{\mathbf{F}_{p^{2}}} \mathbf{F}_{q^{2}} \simeq H^{0}\left(X, \Omega^{1} / \mathbf{F}_{q^{2}}\right) .
$$

On the other hand, it is easy to see that the representation of $G$ on holomorphic differentials on $X$ is isomorphic to $\operatorname{Sym}^{q-2}(U) \otimes \operatorname{det} U$, where $U$ is the standard three-dimensional representation of $U_{3}(q)$ over $\mathbf{F}_{q^{2}}$. The differentials

$$
\omega_{m, n}=x^{m} y^{n} d\left(x / y^{q}\right) \quad \text { with } 0 \leq m, n \text { and } m+n \leq q-2
$$

give a basis, and are eigenvectors for the torus $T$ of $\mathrm{PU}_{3}(q)$ consisting of transformations $(x, y, z) \mapsto(\alpha x, \beta y, \gamma z)$ with $\alpha^{q+1}=\beta^{q+1}=\gamma^{q+1}=1$. The eigenvalue of this transformation on $\omega_{m, n}$ is equal to $\alpha^{m+1} \beta^{n+1} \gamma^{s+1}$ with $m+n+s=q-2$, and these are the weights in the representation $\operatorname{Sym}^{q-2}(U) \otimes$ $\operatorname{det} U$.

The highest weight in the representation $\operatorname{Sym}^{q-2}$ is equal to $(q-2) \omega_{1}=$ $(p-2) \omega_{1}+(p-1) p \omega_{1}+\cdots+(p-1) p^{f-1} \omega_{1}$. By the theory of irreducible representations of $U_{3}(q)$ in characteristic $p, V_{p} \otimes \mathbf{F}_{q^{2}}$ contains an irreducible factor

$$
\text { 4) } \operatorname{Sym}^{p-2}(U) \otimes \operatorname{Sym}^{p-1}\left(U^{p}\right) \otimes \operatorname{Sym}^{p-1}\left(U^{p^{2}}\right) \otimes \cdots \otimes \operatorname{Sym}^{p-1}\left(U^{p^{f-1}}\right) \otimes \operatorname{det} U
$$

with this highest weight [St2]. The representation $V_{\mathfrak{p}}$ is irreducible over $F_{p^{2}}$ if and only if all its irreducible factors over $F_{q^{2}}$ are conjugate to (14.4). This occurs iff $q=p, p^{2}$ by a dimension count. When $q=p, V_{\mathfrak{p}} \simeq \operatorname{Sym}^{p-2}(U) \otimes$ $\operatorname{det} U$ is an absolutely irreducible Hermitian representation. When $q=p^{2}, V_{\mathrm{p}}$ is the restriction to $\mathbf{F}_{p^{2}}$ of the representation $\operatorname{Sym}^{p-2}(U) \otimes \operatorname{Sym}^{p-1}\left(U^{p}\right) \otimes \operatorname{det} U$ over $\mathbf{F}_{p^{4}} ;$ this is self-dual over $\mathbf{F}_{p^{2}}$.

Corollary 14.5. Let $q=p$ or $p^{2}$ and let $V$ be the globally irreducible representation of $G=\mathrm{PU}_{3}(q)$ over $\mathbf{Q}$ of dimension $2 q(q-1)$. Let $R$ be a maximal order in the quaternion division algebra ramified at $p$ and $\infty$. There are $h_{R} \quad(=1$ or 2) Euclidean $\mathrm{Z}[G]$ lattices $L$ in $V$ up to isomorphism with $\operatorname{End}_{G}(L)=R$. 
These lattices have invariants

$$
\begin{aligned}
& \operatorname{rank}(L)=2 q(q-1), \\
& L^{*}=\left\{\begin{array}{lll}
\mathfrak{p}^{-1} L, & \operatorname{det} L=p^{p(p-1)} & \text { if } q=p, \\
L, & \operatorname{det} L=1 & \text { if } q=p^{2}
\end{array} .\right.
\end{aligned}
$$

A model for the lattices $L$ constructed in Corollary 14.5 has been obtained by Elkies. Let $E$ be a supersingular elliptic curve over $\mathbf{F}_{q^{2}}$ with Frobenius endomorphism equal to $-q$ and $\operatorname{End}(E) \simeq R$, and recall that $X$ is the Fermat curve of exponent $(q+1)$. Then

$$
\begin{aligned}
L & =\operatorname{Mor}_{\mathbf{F}_{q^{2}}}(X, E) / \text { translations } \\
& =\operatorname{Hom}_{\mathbf{F}_{q^{2}}}\left(J_{X}, E\right) .
\end{aligned}
$$

When $q=p$, the Hermitian form $\varphi(v, w)=v \circ^{t} w$ is nondegenerate on $\operatorname{Hom}\left(J_{X}, E\right)$. We have $\varphi(v, v)=\operatorname{deg} v$ where $v: X \rightarrow E$ is the associated covering. Since $X$ has $p^{3}+1$ points over $\mathbf{F}_{p^{2}}$, and $E$ has $(p+1)^{2}$ points over $\mathbf{F}_{p^{2}}$, we have the estimate

$$
\langle v, v\rangle \geq 2(p-1)
$$

for all $v \neq 0$ in $L$. When $p=2, L$ is therefore isomorphic to the $D_{4}$-lattice, and when $p=3, L$ is isomorphic to the Coxeter-Todd lattice of rank 12 .

When $q=p^{2}$, the natural Hermitian form $\varphi(v, w)=v o^{t} w$ on $\operatorname{Hom}\left(J_{X}, E\right)$ gives a bilinear form with determinant $p^{2 q(q-1)}=p^{\operatorname{rank}(L)}$. Hence the form giving a unimodular $L$ is equal to $\varphi / p$, which takes values in $\mathfrak{p}^{-1} R$. In particular, $\varphi(v, v) / p$ is integral, so the degree of any covering $v: X \rightarrow E$ is divisible by $p$ ! Counting points as above, we find

$$
\langle v, v\rangle \geq 2 p
$$

for all $v \neq 0$ in $L$, where $\langle$,$\rangle is the pairing under which L^{*}=L$ is unimodular. When $p=2, L$ is the Leech lattice of rank 24 .

For $q=p^{f}$ arbitrary, one can consider the Mordell-Weil lattice of (14.6); this has an action of $G=\mathrm{PU}_{3}(q)$ through its unipotent cuspidal representation, but the reduction is not irreducible at $\mathfrak{p}$ once $f \geq 3$. The conjecture of Birch and Swinnerton-Dyer for the elliptic curve $E$ over the function field $k=\mathbf{F}_{q^{2}}(X)$, which in this case is a theorem due to Tate [Ta2] and Milne [M], gives the identity:

$$
\operatorname{det} L \cdot \# \Perp(E / k)=q^{p(p-1)},
$$

where $\operatorname{det} L$ is calculated for the Euclidean structure $\langle v, w\rangle=\operatorname{Tr} \varphi(v, w)=$ $v \circ^{t} w+w \circ^{t} v$ and $\Perp(E / k)$ is the Tate-Shafarevitch group.

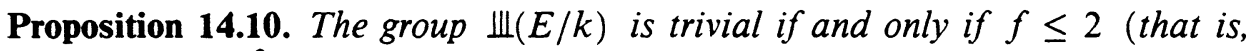

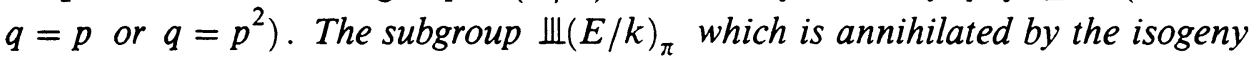
$\pi: E \rightarrow E^{(p)}$ of degree $p$ has order $q^{2 d} / p^{2 g}$, where $d=\frac{1}{4} q(p-1)(q / p+1)$ is the 
dimension of the space of exact holomorphic differentials on $X$ and $g=\frac{1}{2} q(q-1)$ is the genus of $X$.

Proof. By formula (14.9) we see that $\Perp(E / k)$ is a $p$-group, so it is trivial if and only if $\Perp(E / k)_{\pi}=0$. We calculate $\Perp(E / k)_{\pi}$ from a first $\pi$-descent; it is the cokernel of the injection:

$$
E^{(p)}(k) / \pi E(k) \hookrightarrow \operatorname{Sel}_{\pi}(E / k)=H^{1}\left(X, \alpha_{p}\right) .
$$

The first group has order $p^{2 g}$, as it is an $\mathbf{F}_{p^{2}}$ vector space of dimension $g=$ $\frac{1}{4}$ rank $L$. The Selmer group is an $\mathbf{F}_{q^{2}}$ vector space of dimension $d \leq g$. A calculation of the Cartier operator $C$ on $X$ shows that $\omega_{m, n}=x^{m} y^{n} d\left(x / y^{q}\right)$ is exact (that is, $\left.C \omega_{m, n}=0\right)$ if an only if $m \equiv m_{0}, n \equiv n_{0}(\bmod p-1)$ with $0 \leq m_{0}, n_{0} \leq p-1$ and $m_{0}+n_{0} \leq p-2$. This gives the above formula for $d$. Note 14.11. One can show that $C^{f}=0$ on $H^{0}\left(X, \Omega^{1}\right)$, where $q=p^{f}$. The irreducible summand with highest weight $(q-2) \omega_{1}$ described in (14.4) is exactly the image of $C^{f-1}$.

\section{A SUMMARY OF THE EVEN UNIMODULAR LATTICES CONSTRUCTED}

We tabulate the relevant invariants of the even unimodular lattices $L$ we have constructed from globally irreducible representations $V$ in $\S \S 12-14$. In each case, $\operatorname{End}_{G}(V)=K$ is a quaternion algebra ramified in a set $S$ of two rational places. In the following table $p$ is a prime and $n \geq 1$ is an integer.

\begin{tabular}{|c|c|c|c|c|}
\hline$\S$ & $G$ & rank $L$ & $\begin{array}{c}\text { short vectors } \\
v \neq 0 \text { in } L\end{array}$ & $S=$ ramified places \\
\hline 14 & $\mathrm{PU}_{3}\left(p^{2}\right)$ & $2 p^{2}\left(p^{2}-1\right)$ & $\langle v, v\rangle \geq 2 p$ & $\{p, \infty\}$ \\
13 & $\mathrm{Sp}_{2 n}\left(p^{2}\right)$ & $p^{2 n}-1$ & $\langle v, v\rangle \geq \frac{p+1}{2} \quad n=1$ & $\{p, \infty\}$ \\
& $p \neq 2$ & & $\geq ? ? \quad n \geq 2$ & \\
12 & $2^{1+4 n} \cdot O_{4 n}^{-}(2)$ & $2^{2 n+1}$ & $\langle v, v\rangle \geq 2^{n}$ & $\{2, \infty\}$ \\
\hline
\end{tabular}

\section{ACKNOWLEDGMENTS}

It is a great pleasure to thank Noam Elkies for introducing me to the subject of lattices and sphere packings, and for his many helpful suggestions on this paper. I have also profited from conversation and correspondence with E. Bayer, J. Bernstein, W. Feit, J. Humphreys, J. McKay, J.-P. Serre, T. Shioda, J. Thompson, and G. van der Geer.

\section{BIBLIOGRAPHY}

[A] A. Adler, Some integral representations of $\operatorname{PSL}_{2}\left(\mathbf{F}_{p}\right)$ and their applications, J. Algebra 72 (1981), 115-145.

[B] N. Bourbaki, Groupes et algèbres de Lie, Chapitres VII-VIII, Hermann, Paris 1975. 
[BE] M. Broué and M. Enguehard, Une famille infinie de formes quadratiques entières; leurs groupes d'automorphismes, Ann. Sci. École Norm. Sup. 6 (1973), 17-52.

[Ca] R. Carter, Finite groups of Lie type, John Wiley, New York, 1985.

[C] J. H. Conway, et al., ATLAS of finite groups, Clarendon Press, Oxford, 1985.

[CS] J. H. Conway and N. J. A. Sloane, Sphere packings, lattices and groups, Grundlehren Math. Wiss., vol. 290, Springer-Verlag, Berlin and New York, 1988.

[Ei] M. Eichler, Über die Klassenzahl total definiter quaternionenalgebren, Math. Z. 43 (1937), 102-109.

[E] N. Elkies, Letters to N. J. Sloane, 15 August 1989, 15 September 1989.

[F1] W. Feit, The computations of some Schur indices, Israel J. Math. 46 (1983), 274-300.

[F2] — The representation theory of finite groups, North-Holland, Amsterdam, 1982.

[Ge] P. Gérardin, Weil representations associated to finite fields, J. Algebra 46 (1977), 54-101.

[Go1] R. Gow, Schur indices of some groups of Lie type, J. Algebra 42 (1976), 102-120.

[Go2] __ On the Schur indices of characters of finite classical groups, J. London Math. Soc. 24 (1981), 135-147.

[Gr] R. Greiss, Automorphisms of extra-special groups and non-vanishing degree 2 cohomology, Pacific J. Math. 48 (1973), 403-422.

[H] E. Hecke, Über ein fundamentalproblem aus der theorie der Elliptischen modulfunktionen, Abh. Math. Sem. Univ. Hamburg 6 (1928), 235-257 (= Werke 28).

[HM] R. Hotta and K. Matsui, On a lemma of Tate-Thompson, Hiroshima Math. J. 8 (1978), 255-268.

[Hu] J. E. Humphreys, Representations of SL(2, p), Amer. Math. Monthly 82 (1975), 21-39.

[K] G. Karpilovsky, The Schur multiplier, London Math. Soc. Monographs vol. 2, Clarendon Press, Oxford, 1987.

[LS] V. Landazurri and J. Seitz, On the minimal degrees of projective representations of finite Chevalley groups, J. Algebra 32 (1974), 418-443.

[L] G. Lusztig, Coxeter orbits and eigenspaces of Frobenius, Invent. Math. 38 (1976), 101-159.

[Ma] B. Mazur, Arithmetic on curves, Bull. Amer. Math. Soc. 14 (1986), 207-259.

[M] J. Milne, The Tate-Šafarevič group of a constant abelian variety, Invent. Math. 6 (1968), 91-105.

[MH] J. Milnor and D. Husemoller, Symmetric bilinear forms, Ergeb. Math. Grenzgeb., vol. 73, Springer-Verlag, Berlin and New York, 1973.

[Q] D. Quillen, The mod 2 cohomology rings of extra-special 2-groups and the spinor groups, Math. Ann. 194 (1977), 197-212.

[Sch] B. Schoeneberg, Elliptic modular functions, Grundiehren Math. Wiss., vol. 203, SpringerVerlag, Berlin, 1974.

[S1] J.-P. Serre, Complex multiplication, Algebraic Number Theory (J.W.S. Cassels and A. Frohlich, eds.), Academic Press, New York, 1967, pp. 292-296.

[S2] _ A course in arithmetic, Graduate Texts in Math., vol. 7, Springer-Verlag, Berlin and New York, 1973.

[S3] _ Linear representations of finite groups, Graduate Texts in Math., vol. 42, SpringerVerlag, Berlin and New York, 1977.

[Sh1] T. Shioda, Mordell-Weil lattices and Galois representations. I, II, III, Proc. Japan Acad. 65 (1989), 268-271; 296-299; 300-303.

[Sh2] _ Mordell-Weil lattices and sphere packings, preprint, 1989.

[St1] R. Steinberg, Lectures on Chevalley groups, Yale Univ. Press, 1967.

[St2] —-, Representations of algebraic groups, Nagoya Math. J. 22 (1963), 33-56.

[Ta1] J. Tate, Endomorphisms of abelian varieties over finite fields, Invent. Math. 2 (1966), 134144. 
[Ta2] _ On the conjecture of Birch and Swinnerton-Dyer and a geometric analog, Sém. Bourbaki 306 1965-1966.

[Th1] J. G. Thompson, $A$ simple subgroup of $E_{8}(3)$, Finite Groups (N. Iwahori, ed.), Tokyo, 1976, pp. 113-116.

[Th2] _ Finite groups and even lattices, J. Algebra 38 (1976), 523-524.

[V] M.-F. Vigneras, Arithmétique des algèbres de quaternions, Lecture Notes in Math., vol. 800, Springer-Verlag, Berlin and New York, 1980.

[VV] G. van der Geer and M. van der Vlugt, Reed-Muller codes and supersingular curves, Ann. École. Norm. Sup. (to appear).

[ZS] A. E. Zalesskii and I. D. Suprunenko, Representations of dimension $\left(\left(p^{n} \pm 1\right) / 2\right)$ of a symplectic group of degree $2 n$ over a field of characteristic $p$, Vestsī Akad. Navuk BSSR Ser. Fiz.-Math. Navuk 6 (1987), 9-15.

Department of Mathematics, Harvard University, Cambridge, Massachusetts 02138 